\title{
LAPLACE ASYMPTOTICS FOR GENERALIZED K.P.P. EQUATION
}

\author{
JEAN-PHILIPPE ROUQUES
}

\begin{abstract}
Consider a one dimensional non linear reaction-diffusion equation (KPP equation) with non-homogeneous second order term, discontinuous initial condition and small parameter. For points ahead of the Freidlin-KPP front, the solution tends to 0, and we obtain sharp asymptotics (i.e. non logarithmic). Our study follows the work of Ben Arous and Rouault who solved this problem in the homogeneous case. Our proof is probabilistic, and is based on the Feynman-Kac formula and the large deviation principle satisfied by the related diffusions. We use the Laplace method on Wiener space. The main difficulties come from the non-linearity and the possibility for the endpoint of the optimal path to lie on the boundary of the support of the initial condition.
\end{abstract}

\section{INTRODUCTION}

The purpose of this paper is to obtain precise (i.e. non logarithmic) asymptotics of $u^{\varepsilon}(T, x)$ for certain values of $(T, x)$, where $u^{\varepsilon}(T, x)$ is the solution of generalized KPP equation

$$
\left\{\begin{array}{l}
\partial_{t} u^{\varepsilon}=\frac{\varepsilon^{2}}{2} \sigma^{2}(x) \partial_{x x}^{2} u^{\varepsilon}+\frac{c(x)}{\varepsilon^{2}} u^{\varepsilon}\left(1-r\left(u^{\varepsilon}\right)\right) \\
u^{\varepsilon}(0, x)=1_{\{x \leq 0\}}
\end{array}\right.
$$

where $c$ is a non-negative $C^{3}$ function such that there exists $k>0$ satisfying

$$
c(x) \leq k(1+|x|), \quad\left|c^{\prime}(x)\right| \leq k\left(1+x^{2}\right),
$$

$\sigma$ is $C^{2}$, with bounded derivatives, such that there exists $M>m>0$ satisfying

$$
m \leq \sigma(x) \leq M
$$

$r$ is a one-to-one $C^{1}$ increasing function from $[0,1]$ to $[0,1]$.

Our study follows the work of Ben Arous and Rouault (1993) who solved this problem when $\sigma \equiv 1$ and $r(u)=u$.

ESAIM: Probability and Statistics is an electronic journal with URL address http://www.emath.fr/ps/

Submitted January 4, 1996. Revised January 14, 1997. Accepted for publication February 27, 1997

(c) Société de Mathématiques Appliquées et Industrielles. Typeset by $\mathrm{T}_{\mathrm{E}} \mathrm{X}$. 
The Feynman-Kac formula gives an equivalent form of (1.1), that is

$$
u^{\varepsilon}(T, x)=E 1_{\left\{X_{T}^{\varepsilon} \leq 0\right\}} \exp \frac{1}{\varepsilon^{2}} \int_{0}^{T} c\left(X_{s}^{\varepsilon}\right)\left[1-r\left(u^{\varepsilon}\left(T-s, X_{s}^{\varepsilon}\right)\right)\right] d s
$$

where $X^{\varepsilon}$ is the diffusion solution of the stochastic differential equation $X_{t}^{\varepsilon}=x+\varepsilon \int_{0}^{t} \sigma\left(X_{s}^{\varepsilon}\right) d W_{s}$. According to the large deviation principle satisfied by the laws of $\left(X^{\varepsilon}\right)_{\varepsilon>0}$, the limit of $\varepsilon^{2} \ln E\left[f\left(X^{\varepsilon}\right) \exp \varepsilon^{-2} F\left(X^{\varepsilon}\right)\right]$ exists for many continuous functionals $f$ and $F$. By adapting this technique, Freidlin (1985 and 1990) derived the asymptotics of $\ln u^{\varepsilon}(T, x)$ (the difficulty coming from the $u^{\varepsilon}$ in the expectation of (1.4)). He proved the existence of a nonpositive function $V^{*}(T, x)$ such that

i) if $V^{*}(T, x)<0$, then $\varepsilon^{2} \ln u^{\varepsilon}(T, x) \rightarrow V^{*}(T, x)$

ii) if $(T, x)$ is in the interior of $\left\{V^{*}=0\right\}$, then $u^{\varepsilon}(T, x) \rightarrow 1$.

This makes clear the propagation of a wave front: $(t, x)$ is ahead (resp. behind) of the front if $u^{\varepsilon}(t, x) \rightarrow 0$ (resp. $u^{\varepsilon}(t, x) \rightarrow 1$ ).

In order to get precise asymptotics of $E\left[f\left(X^{\varepsilon}\right) \exp \varepsilon^{-2} F\left(X^{\varepsilon}\right)\right]$, one can use the Laplace method on Wiener space (see Doss (1980), Azencott (198081), Ben Arous (1988)) under the standard hypothesis: the maximum of $F-I$ on $\{\psi \mid f(\psi) \neq 0\}$, where $I$ is the action functional of $\left(X^{\varepsilon}\right)_{\varepsilon>0}$, is attained at a unique path $\varphi$, and this maximum is non-degenerate. When $f$ is not continuous at $\varphi$, new techniques are required (see Azencott (1985)). We will use them since the initial condition $1_{1-\infty, 0]}$ is discontinuous at 0 .

However, precise asymptotics of $u^{\varepsilon}(t, x)$ cannot be obtained by using directly Laplace method because of the presence of $u^{\varepsilon}$ in the expectation of (1.4), presence related to the non-linearity of (1.1). Nevertheless, $u^{\varepsilon}(t, x)$ approaches 0 with exponential speed ahead of the front. Thus, if the path $s \rightarrow X_{T-s}^{\varepsilon}(\omega)$ stays ahead of the front, we can neglect most of its contribution; i.e. for all $t<T$ and $\alpha>0$,

$$
\exp -\frac{1}{\varepsilon^{2}} \int_{0}^{t} c\left(X_{s}^{\varepsilon}(\omega)\right) r\left(u^{\varepsilon}\left(T-s, X_{s}^{\varepsilon}(\omega)\right)\right) d s=O\left(\exp -\frac{\alpha}{\varepsilon^{2}}\right)
$$

More precisely, only $\exp -\frac{1}{\varepsilon^{2}} \int_{T-\varepsilon^{a}}^{T} c\left(X_{s}^{\varepsilon}(\omega)\right) r\left(u^{\varepsilon}\left(T-s, X_{s}^{\varepsilon}(\omega)\right)\right) d s$ contributes $(a \in] 0,1\left[; T-\varepsilon^{a}\right.$ and $T$ define a boundary layer, see Ben Arous and Rouault (1993)).

But what happens for other $\omega$ ? Under the Laplace method usual hypothesis, only paths $X^{\varepsilon}(\omega)$ close to the optimal path $\varphi$ really contribute. So, if we want to approximate the case where the paths $s \rightarrow X_{T-s}^{\varepsilon}(\omega)$ stay ahead of the front, we will assume that $\varphi$ stays, in reversed time, ahead of the front (i.e. $\forall s \in\left[0, T\left[V^{*}\left(T-s, \varphi_{s}\right)<0\right)\right.$. This hypothesis is close to condition (N) of Freidlin (see Freidlin (1985 p. 408), and Freidlin (1990) where one can find several examples where this condition is satisfied). A recent result of Barles and Souganidis (1994) concerning the asymptotics of $\varepsilon^{2} \ln \left(1-u^{\varepsilon}(t, x)\right)$ behind the front might enable us to carry the proof to the end without this hypothesis.

In section 2, we state our main results, give connections with branching diffusions and summarize the proof, which starts in section 3 where we 
carry out the Laplace method. We prove in section 4 that only a boundary layer can contribute. In section 5, we construct diffusion bridges using the Skorokhod integral. In section 6 , we study the contribution of the non-linear part and end the proof in section 7 .

\section{Results}

Let $T \in] 0,+\infty\left[\right.$ and $x \in \mathbb{R}$. $H_{x}$ stands for the Cameron-Martin space

$$
\left\{\psi:[0, T] \rightarrow \mathbb{R} \mid \psi_{0}=x, \psi \text { absolutely continuous, } \int_{0}^{T} \dot{\psi}_{s}^{2} d s<\infty\right\}
$$

If $f$ is a continuous function on $[0, T]$, we let $\|f\|=\sup _{0 \leq t \leq T}|f(t)|$. If $f$ is continuous on $[a, b]$, we let $\|f\|_{a}^{b}=\sup _{a<t<b}|f(t)|$. $o(1), O(\varepsilon)$... and positive constants denoted "cst" are all universal, i.e. may only depend on $\sigma, c, r, T$ and $x$.

\subsection{The linear problem}

We introduce the linear problem related to (1.1)

$$
\left\{\begin{array}{l}
\partial_{t} v^{\varepsilon}=\frac{\varepsilon^{2}}{2} \sigma^{2}(x) \partial_{x x}^{2} v^{\varepsilon}+\frac{c(x)}{\varepsilon^{2}} v^{\varepsilon} \\
v^{\varepsilon}(0, x)=1_{\{x \leq 0\}}
\end{array}\right.
$$

Precise asymptotics of $v^{\varepsilon}$ will help computing the ones of $u^{\varepsilon}$ since $u^{\varepsilon} \leq v^{\varepsilon}$ (consequence of the maximum principle or of the Feynman-Kac formula). Let

$$
F(\psi)=\int_{0}^{T} c\left(\psi_{s}\right) d s .
$$

According to the Feynman-Kac formula,

$$
v^{\epsilon}(T, x)=E 1_{\left\{X_{T}^{\epsilon} \leq 0\right\}} \exp \varepsilon^{-2} F\left(X^{\epsilon}\right) .
$$

The laws of $\left(X_{s}^{\varepsilon}\right)_{s \leq T}$ satisfy a large deviation principle with action functional

$$
\begin{aligned}
I(\psi) & =\frac{1}{2} \int_{0}^{T} \dot{\psi}_{s}^{2} S\left(\psi_{s}\right) d s \text { if } \psi \in H_{x} \\
& =+\infty \text { otherwise, }
\end{aligned}
$$

where $S=\sigma^{-2}$. Therefore, $\lim _{\varepsilon \rightarrow 0} \varepsilon^{2} \ln v^{\varepsilon}(T, x)=V(T, x)$, where

$$
V(T, x)=\sup \left\{F(\psi)-I(\psi) \mid \psi \in H_{x}, \psi_{T} \leq 0\right\} .
$$

We will obtain asymptotics of $v^{\varepsilon}(T, x)$ under the Laplace method usual hypothesis:

(H1) the maximum in $V(T, x)$ is attained at a unique path $\varphi$;

(H2) $\varphi$ is a non-degenerate maximum. 
Let $R=F-I$. For $\psi \in H_{x}$ and $h \in H_{0}$,

$$
R^{\prime}(\psi) . h=\int_{0}^{T}\left[c^{\prime}\left(\psi_{s}\right)+\frac{1}{2} \dot{\psi}_{s}^{2} S^{\prime}\left(\psi_{s}\right)+\ddot{\psi}_{s} S\left(\psi_{s}\right)\right] h_{s} d s-\dot{\psi}_{T} S\left(\psi_{T}\right) h_{T} .
$$

Therefore, (H1) yields:

$$
\begin{aligned}
& c^{\prime}(\varphi)+\frac{1}{2} \dot{\varphi}^{2} S^{\prime}(\varphi)+\ddot{\varphi} S(\varphi)=0 \quad \text { (Euler equation) } \\
& -\dot{\varphi}_{T} \geq 0 \\
& \varphi_{T} \dot{\varphi}_{T}=0 \quad \text { (complementary slackness) } \\
& R^{\prime}(\varphi) \cdot h=-\dot{\varphi}_{T} S\left(\varphi_{T}\right) h_{T} \quad \text { for } h \in H_{0} .
\end{aligned}
$$

The case $\varphi_{T}<0$ can be reduced to a problem without constraint and the result is known (see Azencott (1980-81) and Ben Arous (1988)). We will hence study the case $\varphi_{T}=0$.

REMARK 2.1. (2.4) has a geometrical interpretation: since $R$ attains its maximum on $E=\left\{\psi \mid \psi_{T} \leq 0\right\}$ on the boundary of $E$, its gradient at $\varphi$ and the outwardly normal of $E$ in $\varphi$ are positively linked.

We will now focus on the meaning of (H2). We have:

$$
R^{\prime \prime}(\varphi) \cdot h^{2}=\int_{0}^{T}\left[c^{\prime \prime}\left(\varphi_{s}\right)+\frac{1}{2} \dot{\varphi}_{s}^{2} S^{\prime \prime}\left(\varphi_{s}\right)+\ddot{\varphi}_{s} S^{\prime}\left(\varphi_{s}\right)\right] h_{s}^{2} d s-\dot{\varphi}_{T} S^{\prime}(0) h_{T}^{2}-\langle h, h\rangle
$$

where $\langle$,$\rangle is the scalar product defined on H_{0}$ by $\langle h, h\rangle=\int_{0}^{T} \dot{h}_{s}^{2} S\left(\varphi_{s}\right) d s$.

We will say that $\varphi$ is a non-degenerate maximum if there exists $\lambda>0$ such that

$$
R^{\prime \prime}(\varphi) \cdot h^{2} \leq-\lambda\langle h, h\rangle \quad \text { for all } h \in H_{0} .
$$

Introduce now $A$, the self-adjoint Hilbert-Schmidt operator on $H_{0}$ defined by

$$
\langle A h, h\rangle=\int_{0}^{T}\left[c^{\prime \prime}\left(\varphi_{s}\right)+\frac{1}{2} \dot{\varphi}_{s}^{2} S^{\prime \prime}\left(\varphi_{s}\right)+\ddot{\varphi}_{s} S^{\prime}\left(\varphi_{s}\right)\right] h_{s}^{2} d s-\dot{\varphi}_{T} S^{\prime}(0) h_{T}^{2} .
$$

Since $A$ is self-adjoint and compact, there exists a basis $\left(f_{n}\right)_{n>1}$ of eigenvectors of $A$, orthonormal with respect to $\langle$,$\rangle . Define \left(\lambda_{n}\right)_{n>1}$ the corresponding eigenvalues, $\lambda_{1}$ being the largest one. Condition (2.5) is thus equivalent to

$$
\lambda_{1}<1 \text {. }
$$

We now define a gaussian process and its corresponding bridge by

$$
g_{1}(t)=\int_{0}^{t} \sigma\left(\varphi_{s}\right) d W_{s}, \quad g_{1}^{0}(t)=g_{1}(t)-\frac{\int_{0}^{t} \sigma^{2}\left(\varphi_{s}\right) d s}{\int_{0}^{T} \sigma^{2}\left(\varphi_{s}\right) d s} g_{1}(T) t \leq T .
$$

By extending definition (2.6) to continuous functions $h$ on $[0, T]$, we define

$$
K(h)=\frac{1}{2}\langle A h, h\rangle \quad \text { for all } h \in C[0, T] .
$$


Finally, let $p=-\dot{\varphi}_{T} \sigma^{-2}(0)$.

Theorem 2.2. Assume (H1), (H2) (i.e. (2.5) or (2.7)), $\varphi_{T}=0$ and $-\dot{\varphi}_{T}>0$. Then

$$
v^{\varepsilon}(T, x)=\left[A_{1} \varepsilon+o(\varepsilon)\right] \exp \left[\varepsilon^{-2} V(T, x)\right]
$$

where $A_{1}=p^{-1}\left(2 \pi \int_{0}^{T} \sigma^{2}\left(\varphi_{s}\right) d s\right)^{-\frac{1}{2}} E \exp K\left(g_{1}^{0}\right)$.

REMARKS 2.3. i) If $\varphi_{T}<0$, by directly using the Laplace method, we get

$$
v^{\varepsilon}(T, x)=\left[E \exp K\left(g_{1}\right)+o(1)\right] \exp \left[\varepsilon^{-2} V(T, x)\right] .
$$

ii) If $\varphi_{T}=0$ and $-\dot{\varphi}_{T}=0$, it is easy to get (see section 3 )

$$
v^{\varepsilon}(T, x)=\left[E 1_{\left\{g_{1}(T) \leq 0\right\}} \exp K\left(g_{1}\right)+o(1)\right] \exp \left[\varepsilon^{-2} V(T, x)\right] .
$$

The previous constants are finite, as a consequence of Lemma 2.4. There exists $\beta>0$ such that, for all $\alpha \leq \beta$,

$$
E\left[\exp (1+\alpha) K\left(g_{1}\right)\right]<\infty \quad \text { and } \quad E\left[\exp (1+\alpha) K\left(g_{1}^{0}\right)\right]<\infty
$$

Proof. We adapt here a computation of Ben Arous (1988). The equality $\langle A h, h\rangle=\sum_{n=1}^{\infty} \lambda_{n}\left\langle h, f_{n}\right\rangle^{2}$ can be extended by density to continuous martingales $h$ such that $h(0)=0$ if we let $\left\langle h, f_{n}\right\rangle=\int_{0}^{T} \dot{f}_{n}(s) S\left(\varphi_{s}\right) d h_{s}$. Moreover,

$$
E\left[\left\langle g_{1}, f_{n}\right\rangle\left\langle g_{1}, f_{m}\right\rangle\right]=\int_{0}^{T} \dot{f}_{n}(s) S\left(\varphi_{s}\right) \sigma\left(\varphi_{s}\right) \dot{f}_{m}(s) S\left(\varphi_{s}\right) \sigma\left(\varphi_{s}\right) d s=\left\langle f_{n}, f_{m}\right\rangle
$$

Hence, $\left\langle g_{1}, f_{n}\right\rangle$ are independent, gaussian, centered, reduced. Since $A$ is defined by restriction to $H_{0}$ of a continuous quadratic form on $C[0, T], \mathrm{A}$ is a trace class operator $H_{0}$ (see Kuo (1975) th. 4.6 p.83), i.e. $\sum_{n=1}^{\infty}\left|\lambda_{n}\right|<\infty$. Hence, for $(1+\alpha) \lambda_{1}<1$, we have

$$
\begin{aligned}
E\left[\exp (1+\alpha) K\left(g_{1}\right)\right] & =\prod_{n} E\left[\exp \frac{1+\alpha}{2} \lambda_{n}\left\langle g_{1}, f_{n}\right\rangle^{2}\right] \\
& =\prod_{n}\left[1-(1+\alpha) \lambda_{n}\right]^{-\frac{1}{2}} \\
& =(\operatorname{det}[I-(1+\alpha) A])^{-\frac{1}{2}}<\infty .
\end{aligned}
$$

A similar computation with the restriction of $A$ to $\left\{h \in H_{0} / h(T)=0\right\}$ yields $E \exp (1+\alpha) K\left(g_{1}^{0}\right)<\infty$. 


\subsection{The nonlinear problem}

Let

$$
V^{*}(T, x)=\sup \left\{\inf _{0 \leq t \leq T} \int_{0}^{t}\left[c\left(\psi_{s}\right)-\frac{1}{2} \dot{\psi}_{s}^{2} S\left(\psi_{s}\right)\right] d s \mid \psi \in H_{x}, \psi_{T} \leq 0\right\} .
$$

Our first hypothesis is

(H3) i) The maximum in $V^{*}(T, x)$ is attained at a unique path $\varphi$,

ii) $V^{*}\left(s, \varphi_{T-s}\right)<0$ for all $\left.\left.s \in\right] 0, T\right]$,

iii) $V^{*}(T, x)=V(T, x)$.

ii) means that the optimal path $\varphi$ runs always ahead of the front. ii) and iii) are satisfied under condition $(\mathrm{N})$ of Freidlin.

(H3) is equivalent to $V^{*}(T, x)<0$ and the set of $(\psi, t)$ realizing the equality in $V^{*}(T, x)$ is a singleton of the form $(\varphi, T)$. This was proved by Ben Arous and Rouault (1993) in the case $\sigma \equiv 1$, and it can be extended easily.

(H3) yields $x>0, \varphi_{T}=0,-\dot{\varphi}_{T} \geq 0$, and $c(0)-\frac{1}{2} \dot{\varphi}_{T}^{2} S(0) \leq 0$.

We need more than this last inequality to analyse the boundary layer, i.e.

(H4) $-\dot{\varphi}_{T}>\sqrt{2 c(0) \sigma^{2}(0)}$.

(H4) means that $\varphi$, in reversed time, moves quickly away from the front.

Since (H3) implies $V(T, x)=R(\varphi)$, we can select, as a non-degeneracy hypothesis for the nonlinear problem, that of the linear problem (H2).

Finally, let

$$
\begin{aligned}
& g(y)=E \exp -c(0) \int_{0}^{+\infty} r\left(\tilde{u}\left(s,-\dot{\varphi}_{T} s+y+\sigma(0) W_{s}\right)\right) d s \quad \text { where } \\
& \left\{\begin{array}{l}
\partial_{t} \tilde{u}=\frac{1}{2} \sigma^{2}(0) \partial_{x x}^{2} \tilde{u}+c(0) \tilde{u}(1-r(\tilde{u})) \\
\tilde{u}(0, x)=1_{\{x \leq 0\}}
\end{array}\right.
\end{aligned}
$$

Theorem 2.5. Under hypothesis (H2), (H3) and (H4),

$$
u^{\varepsilon}(T, x)=\left[A_{2} \varepsilon+o(\varepsilon)\right] \exp \left[\varepsilon^{-2} V^{*}(T, x)\right]
$$

where $A_{2}=\int_{-\infty}^{0} g(y) \exp p y d y\left(2 \pi \int_{0}^{T} \sigma^{2}\left(\varphi_{s}\right) d s\right)^{-\frac{1}{2}} E \exp K\left(g_{1}^{0}\right)$.

Remarks 2.6. i) If $\sigma \equiv 1$, we can weaken the hypothesis on $r: r$ is a one-to-one increasing continuous function from $[0,1]$ to $[0,1], C^{1}$ on $\left.] 0,1\right]$, $\lim _{u \rightarrow 0} u r^{\prime}(u)=0$ and

$$
\exists \theta>0 \quad \int_{0}^{1} \frac{r(u)}{u^{1+\theta}} d u<+\infty .
$$

ii) By using transformations $x \rightarrow x-a$ or $x \rightarrow-x+a$, we get similar results with initial condition $f(x)=1_{\{x \leq a\}}$ or $f(x)=1_{\{x \geq a\}}$. 
We can also treat the case $f(x)=0$ if $x \geq 0, f(x) \in] 0,1]$ if $x<0$ and $f$ smooth.

Let $k=\inf \left\{n \geq 1 \mid f^{(n)}(0) \neq 0\right\}$. We have

$$
u^{\varepsilon}(T, x)=\left[A_{3} \varepsilon^{k+1}+o\left(\varepsilon^{k+1}\right)\right] \exp \left[\varepsilon^{-2} V^{*}(T, x)\right]
$$

where $A_{3}=\frac{f^{(k)}(0)}{k !} \int_{-\infty}^{0} y^{k} g(y) \exp p y d y\left(2 \pi \int_{0}^{T} \sigma^{2}\left(\varphi_{s}\right) d s\right)^{-\frac{1}{2}} E \exp K\left(g_{1}^{0}\right)$.

iii) Let us prove that $g(y)>0$ for all $y$, and therefore $A_{2}>0$.

Let $\alpha \in] \sqrt{2 c(0) \sigma^{2}(0)},-\dot{\varphi}_{T}[$. We have a.s.

$$
\exists s_{0} \geq 0 \forall s \geq s_{0}-\dot{\varphi}_{T} s+y+\sigma(0) W_{s} \geq \alpha s .
$$

Since $\tilde{u}(s,$.$) is non increasing (Kolmogorov, Petrovskii and Piscunov(1937)),$ we get a.s.

$$
\forall s \geq s_{0} \quad \tilde{u}\left(s,-\dot{\varphi}_{T} s+y+\sigma(0) W_{s}\right) \leq \tilde{u}(s, \alpha s) .
$$

Since $\alpha>\sqrt{2 c(0) \sigma^{2}(0)}$, we have (see Freidlin (1985)),

$$
\lim _{s \rightarrow+\infty} \frac{\ln \tilde{u}(s, \alpha s)}{s}=-\frac{1}{2}\left(\frac{\alpha^{2}}{\sigma^{2}(0)}-2 c(0)\right) .
$$

Therefore, $\int_{0}^{+\infty} r\left(\tilde{u}\left(s,-\dot{\varphi}_{T} s+y+\sigma(0) W_{s}\right)\right) d s<\infty \quad$ a.s. and $g(y)>0$.

\subsection{Connection With BRanching Diffusions}

Let $\lambda$ be a non-negative function on $\mathbb{R}$. Consider the following branching diffusion:

i) a particle starts from $x \in \mathbb{R}$, and executes a small diffusion

$$
d X_{s}^{\varepsilon}=\varepsilon \sigma\left(X_{s}^{\varepsilon}\right) d W_{s}
$$

ii) its lifetime $\tau$ is given by

$$
P\left(\tau \in \left[t, t+d t\left[\mid X_{t}^{\varepsilon}=y, \tau \geq t\right)=\varepsilon^{-2} \lambda(y) d t+o(d t)\right.\right.
$$

iii) when it dies, it is replaced by a random number of descendants $N$

iv) each descendant, starting from where its parent died, repeats i), ii), iii). All diffusions, lifetimes and number of descendants are independent of one another.

Let $N_{t}^{e}$ be the number of particles in $\left.]-\infty, 0\right]$ at time $t$.

Then, $P_{x}\left(N_{t}^{\varepsilon} \neq 0\right)$ is the solution of

$$
\left\{\begin{array}{l}
\partial_{t} u^{\varepsilon}=\frac{\varepsilon^{2}}{2} \sigma^{2}(x) \partial_{x x}^{2} u^{\varepsilon}+\frac{c(x)}{\varepsilon^{2}} f\left(u^{\varepsilon}\right) \\
u^{\varepsilon}(0, x)=1_{\{x \leq 0\}}
\end{array}\right.
$$


where $c(x)=(E N-1) \lambda(x)$ and $f(u)=(E N-1)^{-1}\left(1-u-E(1-u)^{N}\right)$. Assume $P(N=0)=0$ and $E N>1$. Then $f$ is a KPP type non-linearity, i.e.

$$
\begin{aligned}
& f(0)=f(1)=0, \quad f(u)>0 \text { for } u \in] 0,1[ \\
& \left.\left.f \in C^{1}[0,1], \quad f^{\prime}(u)<f^{\prime}(0) \text { for } u \in\right] 0,1\right] .
\end{aligned}
$$

Define $r$ such that $f(u)=u(1-r(u))$. Then $r$ is increasing continuous one-to-one from $[0,1]$ to $[0,1], C^{1}$ on $\left.] 0,1\right]$, and $\lim _{u \rightarrow 0} u r^{\prime}(u)=0$.

The additional hypothesis made on $r$ can be expressed in terms of $N$ : - $r$ is $C^{1}$ on $[0,1]$ if and only if $E\left(N^{2}\right)<\infty$ (easy check).

- (2.10) holds if and only if $E\left(N(\log N)^{1+\theta}\right)<\infty$ (see Athreya and Ney (1972) p. 26).

In the homogeneous case ( $\sigma$ and $c$ constant), Chauvin and Rouault (1988, th.1) obtained asymptotics of $P_{x}\left(N_{t}^{\varepsilon} \neq 0\right)$ for $(t, x)$ in the sub-critical area (i.e. ahead of the front) under the weaker condition $E(N \log N)<\infty$.

We can translate our results into branching diffusions language: since $E_{x} N_{t}^{\varepsilon}$ is solution of linear equation (2.1), we get, under hypothesis of th. 2.1 and 2.3 , asymptotics for $E_{x} N_{T}^{\varepsilon}$ and $P_{x}\left(N_{T}^{\varepsilon} \neq 0\right)$. For instance, $E_{x}\left[N_{T}^{\varepsilon} \mid\right.$ $\left.N_{T}^{\varepsilon} \neq 0\right]$ goes to a finite limit. This means that, when $\left.]-\infty, 0\right]$ is visited at time $T$ (a rare event), the average number of particles in this area is finite.

\subsection{Summary of the proof}

Starting with (2.2) and (1.4), we implement the Laplace method (section 3) which consists in localizing around $\varphi$, applying the Girsanov formula, then performing a stochastic Taylor expansion of the diffusion $Z_{t}^{\varepsilon}=\varepsilon \int_{0}^{t} \sigma\left(\varphi_{s}+\right.$ $\left.Z_{s}^{\varepsilon}\right) d W_{s}$ of the form $Z^{\varepsilon}=\varepsilon g_{1}+\varepsilon^{2} g_{2}+$ remainder ( $g_{1}$ is gaussian). We get $v^{\varepsilon}(T, x) \sim v_{1}^{\varepsilon} \exp \left[\varepsilon^{-2} V(T, x)\right]$ and $u^{\varepsilon}(T, x) \sim u_{1}^{\varepsilon} \exp \left[\varepsilon^{-2} V^{*}(T, x)\right]$ where

$$
\begin{aligned}
& v_{1}^{\varepsilon}=E 1_{\left\{Z_{T}^{\varepsilon} \leq 0,\left\|Z^{\varepsilon}\right\| \leq \rho\right\}} \exp \left[p\left(\varepsilon^{-1} g_{1}(T)+g_{2}(T)\right)+K\left(g_{1}\right)\right] \\
& u_{1}^{\varepsilon}=E 1_{\left\{Z_{T}^{\varepsilon} \leq 0,\left\|Z^{\varepsilon}\right\| \leq \rho\right\}} \\
& \quad \exp \left[p\left(\varepsilon^{-1} g_{1}(T)+g_{2}(T)\right)+K\left(g_{1}\right)-\varepsilon^{-2} F_{\varepsilon}\left(0, T, \varphi+Z^{\varepsilon}\right)\right] \\
& F_{\varepsilon}\left(t, t^{\prime}, \psi\right)=\int_{t}^{t^{\prime}} c\left(\psi_{s}\right) r\left(u^{\varepsilon}\left(T-s, \psi_{s}\right)\right) d s .
\end{aligned}
$$

For $v_{1}^{\varepsilon}$, we now use the following strategy.

i) We prove $v_{1}^{\varepsilon}=E \Psi_{1}^{\varepsilon}\left(g_{1}, g_{2}\right)+o(\varepsilon)$ for some $\Psi_{1}^{\varepsilon}$ (lemma 7.1).

ii) We construct a process $\Delta$ independent of $g_{1}(T)$ such that $g_{1}$ and $g_{2}$ can be expressed in terms of $\Delta$ and $g_{1}(T)$ (lemma 5.1).

iii) Therefore $v_{1}^{\varepsilon}=E \Psi_{2}^{\varepsilon}\left(\Delta, g_{1}(T)\right)+o(\varepsilon)$ for some $\Psi_{2}^{\varepsilon}$. We condition on $\Delta$ and prove that the gaussian integral $\varepsilon^{-1} E \Psi_{2}^{\varepsilon}\left(\delta, g_{1}(T)\right)$ goes to a non zero finite limit (for fixed $\delta$ ). This implies $v_{1}^{\varepsilon} \sim \operatorname{cst} \varepsilon$ (section 7.1).

Concerning the nonlinear problem, we prove in section 4 that we can neglect $F_{\varepsilon}\left(0, T-\varepsilon^{a}, \varphi+Z^{\varepsilon}\right)$ for $\left.a \in\right] 0,1\left[\right.$, i.e. $u_{1}^{\varepsilon}=u_{2}^{\varepsilon}+o(\varepsilon)$ where

$$
\begin{aligned}
u_{2}^{\varepsilon}= & E 1_{\left\{Z_{T}^{\varepsilon} \leq 0,\left\|Z^{\varepsilon}\right\| \leq \rho\right\}} \\
& \exp \left[p\left(\varepsilon^{-1} g_{1}(T)+g_{2}(T)\right)+K\left(g_{1}\right)-\varepsilon^{-2} F_{\varepsilon}\left(T-\varepsilon^{a}, T, \varphi+Z^{\varepsilon}\right)\right] .
\end{aligned}
$$


For $u_{2}^{\varepsilon}$, the strategy is close to that used for $v_{1}^{\varepsilon}$.

i) We condition on $\sigma\left(Z_{s}^{\varepsilon}, s \leq T-\varepsilon^{d} ; Z_{T}^{\varepsilon}\right)$ for $\left.d \in\right] 0, a[$.

From $F_{\varepsilon}\left(T-\varepsilon^{a}, T, \varphi+Z^{\varepsilon}\right)$ arises a functional of $Z^{\varepsilon}$ conditioned on its final position $Z_{T}^{\varepsilon}$ studied in section 6 .

ii) We prove $u_{2}^{\varepsilon}=E \Psi_{3}^{\varepsilon}\left(g_{1}, g_{2}\right)+o(\varepsilon)$ for some $\Psi_{3}^{\varepsilon}$ (lemma 7.4).

iii) We construct a process $\Delta_{\varepsilon}$ independent of $\left(g_{1}\left(T-\varepsilon^{d}\right), g_{1}(T)\right)$ such that $g_{1}$ and $g_{2}$ can be expressed in terms of $\Delta_{\varepsilon}$ and $\left(g_{1}\left(T-\varepsilon^{d}\right), g_{1}(T)\right.$ ) (lemma $5.3)$.

iv) Therefore $u_{2}^{\varepsilon}=E \Psi_{4}^{\varepsilon}\left(\Delta_{\varepsilon}, g_{1}\left(T-\varepsilon^{d}\right), g_{1}(T)\right)+o(\varepsilon)$ for some $\Psi_{4}^{\varepsilon}$. We condition on $\Delta_{\varepsilon}$ and prove that the gaussian double integral $\varepsilon^{-1} E \Psi_{4}^{\varepsilon}\left(\delta, g_{1}\left(T-\varepsilon^{d}\right)\right.$, $g_{1}(T)$ ) goes to a non zero finite limit (for fixed $\delta$ ). This implies $u_{2}^{\varepsilon} \sim \operatorname{cst} \varepsilon$ (section 7.2).

\section{The Laplace Method}

Let $\rho>0$. By (H1), (1.2) and large deviation arguments, there exists $\tau>0$ such that

$v^{\varepsilon}(T, x)=E\left[1_{\left\{X_{T}^{\varepsilon} \leq 0,\left\|X^{\varepsilon}-\varphi\right\| \leq \rho\right\}} \exp \frac{F\left(X^{\varepsilon}\right)}{\varepsilon^{2}}\right]+O\left[\exp \frac{(V(T, x)-\tau)}{\varepsilon^{2}}\right]$.

Then, we apply the Girsanov formula and get (see Azencott (1980-81) pp. 265-266)

$$
\begin{gathered}
\begin{array}{c}
\left.v^{\varepsilon}(T, x)=E 1_{\left\{Z_{T}^{\varepsilon} \leq 0,\right.}\left\|Z^{\varepsilon}\right\| \leq \rho\right\} \\
+O\left[\exp \varepsilon^{-2}(V(T, x)-\tau)\right]
\end{array} \\
\begin{array}{c}
Z_{t}^{\varepsilon}=\varepsilon \int_{0}^{t} \sigma\left(\varphi_{s}+Z_{s}^{\varepsilon}\right) d W_{s} \\
G\left(Z^{\varepsilon}\right)=\int_{0}^{T} \dot{\varphi}_{s} S\left(\varphi_{s}+Z_{s}^{\varepsilon}\right)\left[d Z_{s}^{\varepsilon}+\frac{1}{2} \dot{\varphi}_{s} d s\right] .
\end{array}
\end{gathered}
$$

$Z^{\varepsilon}$ is known to have the following stochastic Taylor expansion (see Azencott (1980-81) p. 251)

$$
Z^{\varepsilon}=\varepsilon g_{1}+\varepsilon^{2} g_{2}+\varepsilon^{3} \Gamma^{\varepsilon} \quad \text { where }
$$

$g_{1}(t)=\int_{0}^{t} \sigma_{0}(s) d W_{s}, \quad g_{2}(t)=\int_{0}^{t} \sigma_{1}(s) g_{1}(s) d W_{s}, \quad$ and $\quad \sigma_{i}(s)=\sigma^{(i)}\left(\varphi_{s}\right)$.

The remainder $\Gamma^{\varepsilon}$ is such that: $\exists c_{1}, c_{2}>0 \quad \forall \rho \geq \varepsilon>0 \quad \forall r \geq c_{1} \rho^{-1}$

$$
P\left(\left\|Z^{\varepsilon}\right\| \leq \rho, \varepsilon\left\|\Gamma^{\varepsilon}\right\| \geq r\right) \leq \exp -c_{2} r \rho^{-1} \text {. }
$$

(3.3) yields, for fixed $\alpha>0$ and $\rho<c_{2} \alpha^{-1}$,

$$
\sup _{\varepsilon \in] 0, \rho]} E 1_{\left\{\left\|Z^{\varepsilon}\right\| \leq \rho\right\}} \exp \alpha \varepsilon\left\|\Gamma^{\varepsilon}\right\| \leq \operatorname{cst}\left(\alpha, \rho, c_{1}, c_{2}\right) .
$$


Remark 3.1. Azencott inequalities can sometimes be improved since their left-hand side members are non-decreasing function of $\rho$.

Thus, the previous inequality becomes: $\forall \alpha>0 \quad \exists \rho_{1}(\alpha)>0$

$$
\sup _{\varepsilon \leq \rho \leq \rho_{1}(\alpha)} E 1_{\left\{\left\|Z^{\varepsilon}\right\| \leq \rho\right\}} \exp \alpha \varepsilon\left\|\Gamma^{\varepsilon}\right\|<\infty
$$

Let $\bar{Z}^{\varepsilon}=\varepsilon g_{1}+\varepsilon^{2} g_{2}$. By composition of Taylor expansions, we get

$$
\begin{aligned}
& F\left(\varphi+Z^{\varepsilon}\right)-G\left(Z^{\varepsilon}\right)=F(\varphi)+F^{\prime}(\varphi) \cdot \bar{Z}^{\varepsilon}+\frac{\varepsilon^{2}}{2} F^{\prime \prime}(\varphi) \cdot g_{1}^{2}-\bar{G}_{\varepsilon}+\varepsilon^{3} \Lambda_{\varepsilon} \\
& \text { where } \bar{G}_{\varepsilon}=\int_{0}^{T} \dot{\varphi}_{s}\left[S\left(\varphi_{s}\right)+S^{\prime}\left(\varphi_{s}\right) \cdot \bar{Z}_{s}^{\varepsilon}+\frac{\varepsilon^{2}}{2} S^{\prime \prime}\left(\varphi_{s}\right) \cdot g_{1}^{2}(s)\right]\left[d \bar{Z}_{s}^{c}+\frac{1}{2} \dot{\varphi}_{s} d s\right] .
\end{aligned}
$$

The remainder $\varepsilon^{3} \Lambda_{\varepsilon}$ satisfies (see Azencott (1980-81) p. 271 and remark 3.1)

$$
\begin{aligned}
& \forall \alpha>0 \quad \exists \rho_{2}(\alpha)>0 \quad \sup _{\varepsilon \leq \rho \leq \rho_{2}(\alpha)} E 1_{\left\{\left\|Z^{\varepsilon}\right\| \leq \rho\right\}} \exp \alpha \varepsilon\left|\Lambda_{\varepsilon}\right|<\infty \\
& \forall \rho>0 \exists c_{3}(\rho)>0 \forall r \geq c_{3}(\rho) P\left(\left\|Z^{\varepsilon}\right\| \leq \rho,\left|\Lambda_{\varepsilon}\right| \geq r\right) \leq \exp -\frac{r^{2 / 3}}{c_{3}(\rho)}
\end{aligned}
$$

We transform (3.5) by using (2.9) and (2.3) (which is still meaningful when $h$ is a continuous martingale on $[0, T]$ such that $h(0)=0)$. We get

$$
F\left(\varphi+Z^{\varepsilon}\right)-G\left(Z^{\varepsilon}\right)=V(T, x)+R^{\prime}(\varphi) \cdot \bar{Z}^{\varepsilon}+\varepsilon^{2} K\left(g_{1}\right)+\varepsilon^{3} \Lambda_{\varepsilon} .
$$

We can extend the identity $R^{\prime}(\varphi) . h=p h_{T}$ by density to continuous martingales $h$ such that $h(0)=0$. Hence, according to (3.1) and (3.8)

$$
\begin{aligned}
v^{\varepsilon}(T, x)=\exp \left[\frac{V(T, x)}{\varepsilon^{-2}}\right] & E 1_{\left\{Z_{T}^{\varepsilon} \leq 0,\left\|Z^{\varepsilon}\right\| \leq \rho\right\}} \exp \left[\frac{p \bar{Z}_{T}^{\varepsilon}}{\varepsilon^{2}}+K\left(g_{1}\right)+\varepsilon \Lambda_{\varepsilon}\right] \\
+ & O\left[\exp \varepsilon^{-2}(V(T, x)-\tau)\right] .
\end{aligned}
$$

Let $v_{1}^{\varepsilon}=E 1_{\left\{Z_{T}^{\varepsilon} \leq 0,\left\|Z^{\varepsilon}\right\| \leq \rho\right\}} \exp \left[p \bar{Z}_{T}^{\varepsilon} \varepsilon^{-2}+K\left(g_{1}\right)\right]$.

LEMMA 3.2. If $\rho$ is small enough, then

$$
v^{\varepsilon}(T, x)=\left[v_{1}^{\varepsilon}+o\left(v_{1}^{\varepsilon}\right)+o(\varepsilon)\right] \exp \left[\varepsilon^{-2} V(T, x)\right] .
$$

Proof. Let $b \in] 0,1[$.

It is enough to prove $w_{1}^{\varepsilon}=o(\varepsilon)$ and $w_{2}^{\varepsilon}=o\left(v_{1}^{\varepsilon}\right)+o(\varepsilon)$ where

$$
\begin{aligned}
& w_{1}^{\varepsilon}=E 1_{\left\{\left|\Lambda_{\varepsilon}\right| \geq \varepsilon^{b-1}, Z_{T}^{\varepsilon} \leq 0,\left\|Z^{\varepsilon}\right\| \leq \rho\right\}} \exp \left[\frac{p \bar{Z}_{T}^{\varepsilon}}{\varepsilon^{2}}+K\left(g_{1}\right)\right]\left[\exp \left(\varepsilon \Lambda_{\varepsilon}\right)-1\right] \\
& w_{2}^{\varepsilon}=E 1_{\left\{\left|\Lambda_{\varepsilon}\right| \leq \varepsilon^{b-1}, Z_{T}^{\varepsilon} \leq 0,\left\|Z^{\varepsilon}\right\| \leq \rho\right\}} \exp \left[\frac{p \bar{Z}_{T}^{\varepsilon}}{\varepsilon^{2}}+K\left(g_{1}\right)\right]\left[\exp \left(\varepsilon \Lambda_{\varepsilon}\right)-1\right]
\end{aligned}
$$

Lemma $2.2,(3.4),(3.6)$ and Hölder inequality yield: $\forall \alpha \in] 0, \beta\left[\exists \rho_{3}(\alpha)>0\right.$

$$
\sup _{\varepsilon \leq \rho \leq \rho_{3}(\alpha)} E 1_{\left\{\left\|Z^{\varepsilon}\right\| \leq \rho\right\}} \exp (1+\alpha)\left[-p \varepsilon \Gamma_{T}^{\varepsilon}+K\left(g_{1}\right)+\varepsilon\left|\Lambda_{\varepsilon}\right|\right]<\infty,
$$


(see remark 3.1), which implies the very useful result

$$
\sup _{\varepsilon \leq \rho \leq \rho_{3}(\alpha)} E 1_{\left\{\left\|Z^{\varepsilon}\right\| \leq \rho\right\}} \exp (1+\alpha)\left[-p \varepsilon \Gamma_{T}^{\varepsilon}+K\left(g_{1}\right)\right]<\infty .
$$

(3.7) and (3.9) yield: for $\varepsilon \leq \rho \leq \rho_{3}\left(\frac{\beta}{2}\right)$ and $\varepsilon^{b-1} \geq c_{2}(\rho)$,

$$
\begin{aligned}
& \left|w_{1}^{\varepsilon}\right| \leq E 1_{\left\{\varepsilon\left|\Lambda_{\varepsilon}\right| \geq \varepsilon^{b}, \| Z^{\varepsilon}|| \leq \rho\right\}} \exp \left[-p \varepsilon \Gamma_{T}^{\varepsilon}+K\left(g_{1}\right)+\varepsilon\left|\Lambda_{\varepsilon}\right|\right] \\
& =O\left(P\left(\left|\Lambda_{\varepsilon}\right| \geq \varepsilon^{b-1},\left\|Z^{\varepsilon}\right\| \leq \rho\right)^{1-2(2+\beta)^{-1}}\right)=O\left(\exp -\operatorname{cst} \varepsilon^{\frac{2}{3}(b-1)}\right) .
\end{aligned}
$$

Let $\left.w_{3}^{\varepsilon}=E 1\left\{\varepsilon\left|\Lambda_{\varepsilon}\right| \leq \varepsilon^{b}, Z_{T}^{\varepsilon} \leq 0,\left\|Z^{\varepsilon}\right\| \leq \rho\right\}\right\}^{\exp \left[p \bar{Z}_{T}^{\varepsilon} \varepsilon^{-2}+K\left(g_{1}\right)+\varepsilon \Lambda_{\varepsilon}\right]}$.

Then, according to (3.7) and (3.9)

$$
\left|w_{2}^{\varepsilon}\right| \leq w_{3}^{\varepsilon}\left(\exp \varepsilon^{b}-1\right) \text { and } v_{1}^{\varepsilon}-w_{3}^{\varepsilon}=O\left(\exp -c s t \varepsilon^{\frac{2}{3}(b-1)}\right),
$$

and therefore $w_{2}^{c}=o\left(v_{1}^{c}\right)+o(\varepsilon)$.

Let us now deal with the nonlinear problem. Let

$$
u_{1}^{\varepsilon}=E 1_{\left\{Z_{T}^{\varepsilon} \leq 0,\left\|Z^{\varepsilon}\right\| \leq \rho\right\}} \exp \left[\frac{p \bar{Z}_{T}^{\varepsilon}}{\varepsilon^{2}}+K\left(g_{1}\right)-\frac{1}{\varepsilon^{2}} F_{\varepsilon}\left(0, T, \varphi+Z^{\varepsilon}\right)\right]
$$

LEMMA 3.3. If $\rho$ is small enough, then

$$
u^{\varepsilon}(T, x)=\left[u_{1}^{\varepsilon}+o\left(u_{1}^{\varepsilon}\right)+o(\varepsilon)\right] \exp \left[\varepsilon^{-2} V^{*}(T, x)\right] .
$$

Proof. If (H1) holds, the proof for the localization is the same. If not, the large deviation argument is not sufficient any more. Ben Arous and Rouault (1993, appendix 3) proved that we can neglect the contribution of the other maxima if $\sigma \equiv 1$. This proof can be extended easily.

The other computations carried out for $v^{\varepsilon}(T, x)$ are still valid for $u^{\varepsilon}(T, x)$.

\section{THE BOUNDARY LAYER}

This section concerns the nonlinear problem. Let $a \in] 0,1[$, and $T(\varepsilon)=$ $T-\varepsilon^{a}$. Ben Arous and Rouault (1993) proved that, when $\sigma \equiv 1$ and $r(u)=u$, (H3ii) and (H4) allow us to neglect $F_{\varepsilon}\left(0, T(\varepsilon), \varphi+Z^{\varepsilon}\right)$ in (3.11). The proof can be extended easily. We will not deal with it in detail. Let $\bar{f}(\eta)=$ $\sup |f(y)|$ for any function $f$.

$|y| \leq \eta$

Lemma 4.1. Let $\eta>0$. Then,

$$
\bar{\sigma}(\eta) \sqrt{2(\bar{c}(2 \eta)+\eta)} s \leq y \leq \bar{\sigma}(\eta) \eta M^{-1} \Longrightarrow u^{\varepsilon}(s, y) \leq 4 \exp -\eta s \varepsilon^{-2} .
$$

Proof. We apply the strong Markov property in (1.4) with $\tau=\inf \{u \leq s \mid$ $\left.\left|X_{u}^{\varepsilon}\right|>\eta\right\}$ 
Lemma 4.2. For $\rho$ small enough, $u_{1}^{\varepsilon}=u_{2}^{\varepsilon}+o(\varepsilon)$ where

$u_{2}^{\varepsilon}=E 1_{\left\{Z_{T}^{\varepsilon} \leq 0,\left\|Z^{\varepsilon}\right\| \leq \rho\right\}} \exp \left[p \bar{Z}_{T}^{\varepsilon} \varepsilon^{-2}+K\left(g_{1}\right)-\varepsilon^{-2} F_{\varepsilon}\left(T(\varepsilon), T, \varphi+Z^{\varepsilon}\right)\right]$.

Proof. For $\delta>0$, let us define the event

$G=\left\{\bar{\sigma}(\eta) \sqrt{2(\bar{c}(2 \eta)+\eta)}(T-s) \leq \varphi_{s}+Z_{s}^{\varepsilon} \leq \frac{\bar{\sigma}(\eta) \eta}{M}, \forall s \in[T-\delta, T(\varepsilon)]\right\}$

(H4) yields the existence of $\rho, \delta, \eta$ such that, for $\varepsilon$ small enough,

$$
P\left((\Omega \backslash G) \cap\left\{\left\|X^{\varepsilon}\right\| \leq \rho\right\}\right)=O\left(\exp -\operatorname{cst} \varepsilon^{a-2}\right)
$$

Thus, it is sufficient to find an upper bound for

$$
\begin{aligned}
& w^{\varepsilon}=E{ }^{1} G \cap\left\{\left\|X^{\varepsilon}\right\| \leq \rho\right\}^{1}\left\{Z_{T}^{\varepsilon} \leq 0,\left\|Z^{\varepsilon}\right\| \leq \rho\right\} \\
& {\left[1-\exp -\varepsilon^{-2} F_{\varepsilon}\left(0, T(\varepsilon), \varphi+Z^{\varepsilon}\right)\right] \exp \left[p \bar{Z}_{T}^{\varepsilon} \varepsilon^{-2}+K\left(g_{1}\right)\right] .}
\end{aligned}
$$

According to lemma 4.1, on $G$,

$$
\begin{aligned}
F_{\varepsilon}\left(T-\delta, T(\varepsilon), \varphi+Z^{\varepsilon}\right) & \leq \bar{c}(\eta) \int_{\varepsilon^{a}}^{\delta} r\left(4 \exp -\eta s \varepsilon^{-2}\right) d s \\
& =\varepsilon^{2} \bar{c}(\eta) \eta^{-1} \int_{4 \exp -\eta \delta \varepsilon^{-2}}^{4 \exp -\eta \varepsilon^{-2+a}} \frac{r(u)}{u} d u \\
& \leq\left\{\begin{array}{l}
c s t \varepsilon^{2} \bar{c}(\eta) \eta^{-1} \exp -\eta \varepsilon^{-2+a} \text { if } r \text { is } C^{1} \text { on }[0,1] \\
\operatorname{cst} \varepsilon^{2} \bar{c}(\eta) \eta^{-1} \exp -\theta \eta \varepsilon^{-2+a} \text { if }(2.10) \text { holds. }
\end{array}\right.
\end{aligned}
$$

(H3ii) yields, on $\left\{\left\|X^{\varepsilon}\right\| \leq \rho\right\}$ and for $s \in[0, T-\delta]$,

$$
\begin{aligned}
u^{\varepsilon}\left(s, \varphi_{s}+Z_{s}^{\varepsilon}\right) & \leq \exp -\operatorname{cst} \varepsilon^{-2} \\
F_{\varepsilon}\left(0, T-\delta, \varphi+Z^{\varepsilon}\right) & \leq \operatorname{cst} r\left(\exp -\operatorname{cst} \varepsilon^{-2}\right) \\
& \leq\left\{\begin{array}{l}
\text { cst } \exp -\operatorname{cst} \varepsilon^{-2} \text { if } r \text { is } C^{1} \text { on }[0,1] \\
\operatorname{cst} \varepsilon^{4} \text { if }(2.13) \text { holds. }
\end{array}\right.
\end{aligned}
$$

Actually, the fact that $r$ is non-decreasing and (2.13) imply:

$r\left(\exp -\varepsilon^{-1}\right)=O\left(\varepsilon^{\mu}\right)$ for all $\mu>0$. Therefore, according to (4.1) and (4.2), $F_{\varepsilon}\left(0, T(\varepsilon), \varphi+Z^{\varepsilon}\right)=o\left(\varepsilon^{3}\right)$, and

$$
w^{\varepsilon} \leq o(\varepsilon) E 1_{\left\{\left\|Z^{\varepsilon}\right\| \leq \rho\right\}} \exp \left(-p \varepsilon \Gamma_{T}^{\varepsilon}+K\left(g_{1}\right)\right)
$$

We conclude by using (3.10). 


\section{CONSTRUCTION OF DifFusion BRIDGES}

To understand the asymptotics of $v_{1}^{\varepsilon}$ and $u_{2}^{\varepsilon}$, Ben Arous and Rouault ((1993) pp. 272, 274) introduced the brownian bridge $W^{0}=\left(W_{s}-s T^{-1} W_{T}\right)_{0 \leq s \leq T}$, and used independence between $W^{0}$ and $W_{T}$. In this section, we construct "bridges" associated to the non gaussian diffusion $\left(g_{1}, g_{2}\right)$.

For $\alpha, \lambda, \mu>0$, define $\mathcal{E}(\alpha, \lambda, \mu)$ to be the set of continuous processes $X$ such that

$$
\forall r \geq 0 \quad P(\|X\| \geq r) \leq \lambda \exp -\mu r^{\alpha} .
$$

Let $\mathcal{E}=\cup_{\alpha, \lambda, \mu>0} \mathcal{E}(\alpha, \lambda, \mu)$ and $Y=-g_{1}(T)$.

Lemma 5.1. Construction of a single bridge $\Delta$

There exists a process $\Delta=\left\{G_{10}, G_{20}, G_{21}\right\}$, independent of $Y$, whose components are in $\mathcal{E}$, and there exist $G_{11}$ and $G_{22}$ in $C^{1}[0, T]$ such that $G_{10}=g_{1}^{0}$ and

$$
g_{1}=G_{10}+G_{11} Y, \quad g_{2}=G_{20}+G_{21} Y+G_{22} Y^{2} .
$$

Proof. Let $a_{t}=-\left(\int_{0}^{T} \sigma_{0}^{2}(s) d s\right)^{-1} \int_{0}^{t} \sigma_{0}(s) d s$ and $\widetilde{W}_{t}=W_{t}-a_{t} Y$ for $t \in[0, T]$. The gaussian process $\widetilde{W}$ is independent of $Y$.

Then, define $\int_{0}^{t} X_{s} d \widetilde{W}_{s}=\int_{0}^{t} X_{s} d W_{s}-Y \int_{0}^{t} X_{s} d a_{s}$ for $X$ a continuous and adapted process of $L^{2}(\Omega \times[0, T])$. We have,

$$
\begin{aligned}
& g_{1}=G_{10}+G_{11} Y, \quad G_{10}(.)=\int_{0}^{\cdot} \sigma_{0}(s) d \widetilde{W}_{s}, \quad G_{11}(.)=\int_{0} \sigma_{0}(s) d a_{s} \\
& g_{2}(.)=\int_{0} \sigma_{1}(s)\left[G_{10}(s)+G_{11}(s) Y\right] d W_{s} .
\end{aligned}
$$

Since $G_{10}$ and $G_{11} Y$ are not adapted to the filtration of $W$, we cannot develop this previous expression of $g_{2}$ by linearity. So we use the Skorokhod integral which extends Itô integral and accepts non-adapted integrands. $\int_{0}^{t} X_{s} \delta W_{s}$ stands for the Skorokhod integral of $X \in \mathrm{dom} \delta$. Define $\delta \widetilde{W}_{s}$ in the natural way. We have,

$$
\begin{aligned}
g_{2}(.)= & \int_{0} \sigma_{1}(s) G_{10}(s) \delta W_{s}+\int_{0}^{\cdot} \sigma_{1}(s) G_{11}(s) Y \delta W_{s} \\
= & \int_{0} \sigma_{1}(s) G_{10}(s) \delta \widetilde{W}_{s}+Y \int_{0} \sigma_{1}(s) G_{10}(s) d a_{s} \\
& \quad+\int_{0} Y \sigma_{1}(s) G_{11}(s) \delta \widetilde{W}_{s}+Y^{2} \int_{0} \sigma_{1}(s) G_{11}(s) d a_{s} .
\end{aligned}
$$

We know that (see for instance Nualart and Pardoux (1988)),

$$
\begin{aligned}
& \int_{0}^{t} Y \sigma_{1}(s) G_{11}(s) \delta W_{s}= \\
& \quad Y \int_{0}^{t} \sigma_{1}(s) G_{11}(s) d W_{s}-\int_{0}^{t} \sigma_{1}(s) G_{11}(s) D_{s} Y d s
\end{aligned}
$$


where $D_{s}$ stands for the Malliavin derivative. Since $D_{s} Y=-\sigma_{0}(s)$, we get

$$
\begin{aligned}
& g_{2}=G_{22} Y^{2}+G_{21} Y+G_{20}, \quad G_{22}(.)=\int_{0}^{\cdot} \sigma_{1}(s) G_{11}(s) d a_{s} \\
& G_{20}(.)=\int_{0}^{\cdot} \sigma_{1}(s) G_{10}(s) \delta \widetilde{W}_{s}+\int_{0}^{\cdot} \sigma_{0}(s) \sigma_{1}(s) G_{11}(s) d s \\
& G_{21}(.)=\int_{0}^{\cdot} \sigma_{1}(s) G_{10}(s) d a_{s}+\int_{0}^{\cdot} \sigma_{1}(s) G_{11}(s) \delta \widetilde{W}_{s} .
\end{aligned}
$$

Let $\mathcal{H}_{m}$ be the $\mathrm{m}^{\text {th }}$ Wiener chaos.

Lemma 5.2. Let $X=\left(X^{1}, \ldots, X^{n}\right)$ be a continuous $\mathbb{R}^{n}$-valued process.

Assume

i) $(X, \widetilde{W})$ and $Y$ are independent

ii) $\forall i \in\{1, \ldots, n\} \forall s \in[0, T] X_{s}^{i} \in \mathcal{H}_{0} \oplus \mathcal{H}_{1}$.

Then, $\left(\int_{0} X_{s} \delta \widetilde{W}_{s}, \widetilde{W}\right)$ and $Y$ are independent.

Lemma 5.2. is proved below. Lemma 5.2 with $X=\sigma_{0}$ yields independence between $\left(G_{10}, \widetilde{W}\right)$ and $Y$. Then, with $X=\left(\sigma_{0}, \sigma_{1} G_{10}, \sigma_{1} G_{11}\right)$, it yields that $\left(G_{10}, G_{20}, G_{21}\right)$ and $Y$ are independent.

It remains to prove that $G_{10}, G_{20}$ and $G_{21}$ belong to $\mathcal{E}$. For $G_{10}$ and $G_{21}$, it is a consequence of the stability of $\mathcal{E}$ under sum, product, Riemann and Itô integration (see Azencott (1980-81) p. 252). For $G_{20}$, we just need to prove $\int_{0} \sigma_{1}(s) G_{10}(s) \delta W_{s} \in \mathcal{E}$. The space $\mathcal{E}$ is not stable under Skorokhod integration, but identity $G_{10}=g_{1}-Y G_{11}$ and (5.1) allow us to conclude. $\square$ Proof of lemma 5.2. Let us prove that

$$
\operatorname{Ef}\left(\int_{0}^{t} X_{s} \delta \widetilde{W}_{s}, \widetilde{W}_{t}\right) h(Y)=\operatorname{Ef}\left(\int_{0}^{t} X_{s} \delta \widetilde{W}_{s}, \widetilde{W}_{t}\right) \operatorname{Eh}(Y)
$$

for $t \in[0, T]$ and $f, h$ bounded continuous functions (we only deal with one-dimensional marginals not to overload the notations).

Let $\Pi^{p}: 0=t_{0, p}<\cdots<t_{p, p}=t$ be a sequence of partitions of $[0, t]$ whose meshes go to 0 , and let

$$
X_{p}=\sum_{k=0}^{p-1} \bar{X}_{k, p} 1_{\left[t_{k, p}, t_{k+1, p}[\right.}, \quad \bar{X}_{k, p}=\left(t_{k+1, p}-t_{k, p}\right)^{-1} \int_{t_{k, p}}^{t_{k+1, p}} X_{u} d u .
$$

We have (see Nualart and Pardoux (1988), prop. 4.3 and remark p. 546)

$$
\int_{0}^{t} X_{p}(s) \delta W_{s} \longrightarrow \int_{0}^{t} X_{s} \delta W_{s} \quad \text { in } L^{2}(\Omega) .
$$

Therefore, there exists a subsequence of $\Pi^{p}$ such that this convergence holds a.s. It yields

$$
Z_{p}:=\int_{0}^{t} X_{p}(s) \delta \widetilde{W}_{s} \longrightarrow \int_{0}^{t} X_{s} \delta \widetilde{W}_{s} \text { a.s. }
$$


By dominated convergence, it is enough to prove that $Z_{p}$ is independent of $Y$.

Since the Malliavin derivative and Riemann integral commute,

$$
\begin{aligned}
& Z_{p}= \sum_{k=0}^{p-1} \bar{X}_{k, p}\left(\widetilde{W}_{t_{k+1, p}}-\widetilde{W}_{t_{k, p}}\right)-\int_{t_{k, p}}^{t_{k+1, p}} D_{s} \bar{X}_{k, p} d s \\
&=\sum_{k=0}^{p-1} \bar{X}_{k, p}\left(\widetilde{W}_{t_{k+1, p}}-\widetilde{W}_{t_{k, p}}\right) \\
& \quad-\left(t_{k+1, p}-t_{k, p}\right)^{-1} \int_{\left[t_{k, p} ; t_{k+1, p}\right]^{2}} D_{s} X_{u} d u d s .
\end{aligned}
$$

But $X_{u}^{i} \in \mathcal{H}_{0} \oplus \mathcal{H}_{1}$ yields that $D_{s} X_{u}$ is deterministic. Therefore, $Z_{p}$ can be expressed in terms of $(X, \widetilde{W})$ which is independent of $Y$.

Let $V_{\varepsilon}=\left(Y_{\varepsilon}, Y\right)=\left(-g_{1}\left(T^{\prime}(\varepsilon)\right),-g_{1}(T)\right)$ where $T^{\prime}(\varepsilon)=T-\varepsilon^{d}$ and $d \in] 0, a[$.

Lemma 5.3. Construction of a double bridge $\Delta_{\varepsilon}$. There exists a process $\Delta_{\varepsilon}=\left\{G_{i j k}^{\varepsilon}, 0 \leq j+k \leq i \leq 2\right\}$, independent of $V_{\varepsilon}$, such that:

i) $g_{i}=\sum_{0 \leq j+k \leq i} G_{i j k}^{\varepsilon} Y_{\varepsilon}^{j} Y^{k}, \quad i=1,2$

ii) If $j+k=i, G_{i j k}^{\varepsilon}$ is a deterministic $C^{1}$ function on $[0, T]$.

iii) $\exists \alpha, \lambda, \mu>0 \forall \varepsilon>0 \forall 0 \leq j+k \leq i \leq 2 \quad G_{i j k}^{\varepsilon} \in \mathcal{E}(\alpha, \lambda, \mu)$.

Proof. Define $\widetilde{W}^{\varepsilon}$, a gaussian process independent of $V_{\varepsilon}$ :

$$
\begin{aligned}
& \widetilde{W}_{t}^{\varepsilon}=W_{t}-b^{\varepsilon}(t) Y_{\varepsilon}-a^{\varepsilon}(t) Y, \quad 0 \leq t \leq T \\
& a_{t}^{\varepsilon}=-1_{\left\{t \geq T^{\prime}(\varepsilon)\right\}}\left(\int_{T^{\prime}(\varepsilon)}^{T} \sigma_{0}^{2}(s) d s\right)^{-1} \int_{T^{\prime}(\varepsilon)}^{t} \sigma_{0}(s) d s \\
& b_{t}^{\varepsilon}=a_{t}^{\varepsilon}-\left(\int_{0}^{T^{\prime}(\varepsilon)} \sigma_{0}^{2}(s) d s\right)^{-1} \int_{0}^{\inf \left(t, T^{\prime}(\varepsilon)\right)} \sigma_{0}(s) d s .
\end{aligned}
$$

Then, let $d \widetilde{W}_{s}^{\varepsilon}=d W_{s}-Y_{\varepsilon} d b^{\varepsilon}(s)-Y d a^{\varepsilon}(s)$. The proof is now similar to that of lemma 5.1. The only new point is to prove that the processes $G_{i j k}^{\varepsilon}$ belong to the same $\mathcal{E}(\alpha, \lambda, \mu)$ for all $\varepsilon$. If $j+k=i$, it is clear because $G_{i j k}^{\varepsilon}$ is deterministic and uniformly bounded in $\varepsilon$. If $j+k<i$, it comes from Azencott results (see Azencott (1980-81) p. 252).

Exact expressions of $G_{i j k}^{\epsilon}$ do not matter, except for

$$
G_{100}^{\varepsilon}(.)=\int_{0}^{\cdot} \sigma_{0}(s) d W_{s}^{\varepsilon}
$$

\section{ThE NON-Linear PART CONTRIBUTION}




\subsection{Results}

Let $\alpha \in] 0,1-a\left[\right.$ and $g^{\varepsilon}(y, z)$ given by

$$
E\left[1_{\left\{\left\|Z^{\varepsilon}\right\|_{T(\varepsilon)}^{T} \leq \varepsilon^{1-\alpha}\right\}} \exp -\frac{1}{\varepsilon^{2}} F_{\varepsilon}\left(T(\varepsilon), T, \varphi+Z^{\varepsilon}\right) \mid Z_{T^{\prime}(\varepsilon)}^{\varepsilon}=\varepsilon z, Z_{T}^{\varepsilon}=\varepsilon^{2} y\right]
$$

In this section, we prove $g^{\varepsilon}(y, z) \rightarrow g(y)$, thus understanding the contribution of $u^{\varepsilon}$ in the expectation of (2.11). The key result is lemma 6.1 that establishes the convergence of $u^{\varepsilon}\left(\varepsilon^{2} t, \varepsilon^{2} x\right)$.

If $\sigma \equiv 1$, the equality between the laws of a brownian motion knowing its final position and the related bridge makes the computation more simple (see Ben Arous and Rouault 1993). Our method is based upon the following classical result (see Fitzsimmons, Pitman and Yor (1993)).

Let $\left(X_{t}\right)_{t<T}$ be a real Markov process with transition density $p_{s, t}(x, y)$, and $\left(\mathcal{F}_{t}\right)_{t<T}$ be its natural filtration. Let $x \in \mathbb{R}, t<T$ and $Z$ a r.v. $\mathcal{F}_{t}$-measurable. Then, for almost every $y \in \mathbb{R}$,

$$
E_{x}\left[Z \mid X_{T}=y\right] p_{0, T}(x, y)=E_{x}\left[Z p_{t, T}\left(X_{t}, y\right)\right] \text {. }
$$

In order to use (6.2) and make $u^{\varepsilon}\left(\varepsilon^{2} t, \varepsilon^{2} x\right)$ appear, let $Y_{s}^{\varepsilon}=\varepsilon^{-2} Z_{T-\varepsilon^{2} s}^{\varepsilon}$.

We have

$$
\begin{aligned}
& g^{\varepsilon}(y, z)=E\left[{ }^{1} B_{\varepsilon} \exp -I_{\varepsilon} \mid Y_{\varepsilon^{d-2}}^{\varepsilon}=\varepsilon^{-1} z\right] \\
& Y_{t}^{\varepsilon}=y+\int_{0}^{t} \sigma\left(\varphi_{T-\varepsilon^{2} s}+\varepsilon^{2} Y_{s}^{\varepsilon}\right) d W_{s} \\
& B_{\varepsilon}=\left\{\left\|Y^{\varepsilon}\right\|_{0}^{\varepsilon^{a-2}} \leq \varepsilon^{-1-\alpha}\right\} \\
& I_{\varepsilon}=\int_{0}^{\varepsilon^{a-2}} c\left(\varphi_{T-\varepsilon^{2} s}+\varepsilon^{2} Y_{s}^{\varepsilon}\right) r\left(u^{\varepsilon}\left(\varepsilon^{2} s, \varphi_{T-\varepsilon^{2} s}+\varepsilon^{2} Y_{s}^{\varepsilon}\right)\right) d s .
\end{aligned}
$$

We will prove that, if $z \varepsilon^{-1}$ is not too large, the conditioning on $Y_{\varepsilon^{d-2}}^{\varepsilon}=z \varepsilon^{-1}$ does not alter the asymptotics. Actually, "tying up" a diffusion $Y$ at time $t$ on point $y_{t} \in R$ does not alter $E\left[\Psi\left(Y_{s}, s \leq t^{\prime}\right)\right]$ when $t$ and $t^{\prime}$ go to $+\infty$, provided that $t^{\prime}=o(t)$ and that $y_{t}$ is not too large. Let

$$
g^{\varepsilon}(y)=E\left[1_{B_{\varepsilon}} \exp -I_{\varepsilon}\right]
$$

Proposition 6.1. Assume $2 / 3<d<a<1$. Then

i) For all $y, g^{\varepsilon}(y) \rightarrow g(y)$.

ii) Assume $z=O\left(\varepsilon^{1-\nu}\right)$ where $\left.\nu \in\right] 0,1+\frac{a}{2}-d\left[\right.$. Then, $g^{\varepsilon}(y, z)-g^{\varepsilon}(y) \rightarrow 0$. We need, in section 7 , the following result on the modulus of continuity of $g^{\varepsilon}(y, z)$.

Proposition 6.2. Assume $r \in C^{1}[0,1]$ (case $\sigma$ non constant).

Assume $2 / 3<d<a<1$. Let $\eta>0$ small enough, and $b>0$. Then i) If $\left|y-y^{\prime}\right| \leq \varepsilon^{b}$ and $|y|,\left|y^{\prime}\right| \leq \varepsilon^{-\eta}$, then $g^{\varepsilon}(y)-g^{\varepsilon}\left(y^{\prime}\right)=o(1)$.

ii) If moreover $\left|z-z^{\prime}\right| \leq \varepsilon^{d+\eta}$ and $|z|,\left|z^{\prime}\right| \leq \varepsilon^{\frac{1}{2} d-\eta}$, then $g^{\varepsilon}(y, z)-g^{\varepsilon}\left(y^{\prime}, z^{\prime}\right)=$ $o(1)$.

REMARK 6.3. We state propositions 6.1 .i and $6.2 . \mathrm{i}$ to show that the conditioning has no influence and to make the proofs easier to read. 


\subsection{Proof of proposition 6.1.I.}

Lemma 6.4. Let $t_{0}>0$. Then $u^{\varepsilon}\left(\varepsilon^{2} t, \varepsilon^{2} x\right) \rightarrow \tilde{u}(t, x)$ uniformly for $\left.t \in\right] 0, t_{0}[$ and for $x$ such that $\varepsilon^{2} x \rightarrow 0$.

Proof. Since $\left.\left.r \in C^{1}\right] 0,1\right]$ and $\lim _{u \rightarrow 0} u r^{\prime}(u)=0$, there exists a continuous function $\rho$ on $[0,1]^{2}$ such that $\rho(u, v)=\frac{u r(u)-v r(v)}{u-v}$ if $u \neq v$.

Let $h^{\varepsilon}(t, x)=u^{\varepsilon}\left(\varepsilon^{2} t, \varepsilon^{2} x\right)-\tilde{u}(t, x)$. Then $h^{\varepsilon}(0, x)=0$ and

$$
\begin{aligned}
& \partial_{t} h^{\varepsilon}=\frac{\sigma^{2}(0)}{2} \partial_{x x}^{2} h^{\varepsilon}+\alpha_{\varepsilon}+\beta_{\varepsilon} h^{\varepsilon} \\
& \alpha_{\varepsilon}(t, x)=\frac{1}{2}\left(\sigma^{2}\left(\varepsilon^{2} x\right)-\sigma^{2}(0)\right) \partial_{x x}^{2} u^{\varepsilon}\left(\varepsilon^{2} t, \varepsilon^{2} x\right)+\left(c\left(\varepsilon^{2} x\right)-c(0)\right) \tilde{u}(1-r(\tilde{u})) \\
& \beta_{\varepsilon}(t, x)=c\left(\varepsilon^{2} x\right)\left[1-\rho\left(\tilde{u}^{\varepsilon}(t, x), \tilde{u}(t, x)\right)\right] .
\end{aligned}
$$

Therefore, the Feynman-Kac formula yields

$$
h^{\varepsilon}(t, x)=E \int_{0}^{t} \alpha_{\varepsilon}\left(t-s, x+\sigma(0) W_{s}\right) \exp \left(\int_{0}^{s} \beta_{\varepsilon}\left(t-u, x+\sigma(0) W_{u}\right) d u\right) d s .
$$

(1.2) implies $\left|\beta_{\varepsilon}(t, x)\right| \leq k \sup _{[0,1]^{2}}|1-\rho|\left(1+\varepsilon^{2}|x|\right)$.

Appendix 8.1 implies: $\exists C>0 \quad\left|\partial_{x x}^{2} u^{\varepsilon}\left(\varepsilon^{2} t, \varepsilon^{2} x\right)\right| \leq C\left(\sqrt{t}+t^{2} x^{2}+t^{\frac{5}{2}}\right)$. $(1.2),(1.3)$ and the previous two bounds allow us to end the proof by applying to $(6.5)$ a joint dominated convergence in $\omega$ and $s$.

We come back to the proof of proposition 6.1.i. where

Define $R^{\varepsilon}$ such that $Y_{s}^{\varepsilon}=y+\sigma(0) W_{s}+\varepsilon R_{s}^{\varepsilon}$. Then $d R_{s}^{\varepsilon}=A_{s}^{\varepsilon} d W_{s}$

$$
A_{s}^{\varepsilon}=\varepsilon^{-1}\left(\sigma\left(\varphi_{T-\varepsilon^{2} s}+\varepsilon^{2} Y_{s}^{\varepsilon}\right)-\sigma(0)\right)=O\left(\varepsilon^{-\alpha}\right) \quad \text { on } B_{\varepsilon} \text { and if } s \leq \varepsilon^{-1} .
$$

Therefore $P\left(\left\|R^{\varepsilon}\right\|_{0}^{\varepsilon^{-1}} \geq \varepsilon^{-\delta} ; B_{\varepsilon}\right) \leq \exp -\operatorname{cst} \varepsilon^{1+2 \alpha-2 \delta}$.

Since $\alpha<1-a<\frac{1}{2}$, there exists $\left.\delta \in\right] \frac{1}{2}+\alpha, 1[$. Hence,

$$
g^{\varepsilon}(y)=E\left[1_{\left\{\left\|R^{\varepsilon}\right\|_{0}^{\varepsilon^{-1}} \leq \varepsilon^{-\delta}\right\} \cap B_{\varepsilon}} \exp -I_{\varepsilon}\right]+o(1) .
$$

Let $L=c(0) \int_{0}^{+\infty} r\left(\tilde{u}\left(s,-\dot{\varphi}_{T} s+y+\sigma(0) W_{s}\right)\right) d s$.

Since $P\left(\left\{\left\|R^{\varepsilon}\right\|_{0}^{\varepsilon^{-1}} \leq \varepsilon^{-\delta}\right\} \cap B_{\varepsilon}\right) \rightarrow 1$, proposition 6.1 .i is implied by

$$
{ }^{E 1}\left\{\left\|R^{\varepsilon}\right\|_{0}^{\varepsilon^{-1}} \leq \varepsilon^{-\delta}\right\} \cap B_{\varepsilon}\left[\exp \left(-I_{\varepsilon}\right)-\exp (-L)\right] \rightarrow 0 .
$$

By dominated convergence, it is enough to prove

$$
{ }^{1}\left\{\left\|R^{\varepsilon}\right\|_{0}^{\varepsilon^{-1}} \leq \varepsilon^{-\delta}\right\} \cap B_{\varepsilon}\left(I_{\varepsilon}-L\right) \rightarrow 0 \text { a.s. }
$$

REMARK 6.5. $\varepsilon$ stands for the general term of a sequence going to 0 , thus avoiding non-countability problems. Th. 2.1 and 2.3 can easily be reduced to this case. 
Therefore, we can assume $\omega \in\left\{\left\|R^{\varepsilon}\right\|_{0}^{\varepsilon^{-1}} \leq \varepsilon^{-\delta}\right\} \cap B_{\varepsilon}$ for all $\varepsilon$.

For fixed $s$ and $\varepsilon \leq s^{-1}$,

$$
\left|Y_{s}^{\varepsilon}(\omega)-y-\sigma(0) W_{s}(\omega)\right| \leq \varepsilon^{1-\delta}
$$

Hence, by lemma $6.3, c\left(\varphi_{T-\varepsilon^{2} s}+\varepsilon^{2} Y_{s}^{\varepsilon}(\omega)\right) r\left(u^{\varepsilon}\left(\varepsilon^{2} s, \varphi_{T-\varepsilon^{2} s}+\varepsilon^{2} Y_{s}^{\varepsilon}(\omega)\right)\right)$ goes to $c(0) r\left(\tilde{u}\left(s,-\dot{\varphi}_{T} s+y+\sigma(0) W_{s}(\omega)\right)\right)$ for all $s$.

(6.6) will be deduced, provided that we have a dominated convergence in $s$. Let

$$
\Omega_{1}=\left\{\omega\left|\forall \delta>0 \quad \exists s_{0}(\omega) \quad \forall \varepsilon \quad \forall s \geq s_{0}(\omega)\right| Y_{s}^{\varepsilon}(\omega) \mid \leq \delta s\right\}
$$

(8.8) implies $P\left(\Omega_{1}\right)=1$. Hence we assume $\omega \in \Omega_{1}$.

By lemma 4.1 , for all $\eta>0$,

$$
\bar{\sigma}(\eta) \sqrt{2(\bar{c}(2 \eta)+\eta)} s \leq x \leq \bar{\sigma}(\eta) \eta M^{-1} \varepsilon^{-2} \Longrightarrow \tilde{u}^{\varepsilon}(s, x) \leq 4 \exp -\eta s .
$$

(H4) yields the existence of $\eta>0$ such that $-\dot{\varphi}_{T}>\bar{\sigma}(\eta) \sqrt{2(\bar{c}(2 \eta)+\eta)}+\eta$.

For $s \in\left[0, \varepsilon^{a-2}\right]$ and $\varepsilon$ small enough, $\left|e^{-2} s^{-1} \varphi_{T-\varepsilon^{2} s}+\dot{\varphi}_{T}\right| \leq \frac{\eta}{2}$.

$\omega \in \Omega_{1}$ implies: $\exists s_{0}(\omega) \quad \forall \varepsilon \quad \forall s \geq s_{0}(\omega)\left|Y_{s}^{\varepsilon}(\omega)\right| \leq \frac{\eta}{2} s$.

Hence, according to $(6.7)$, for $s_{0}(\omega) \leq s \leq \varepsilon^{a-2}$ and $\varepsilon$ small enough,

$$
\begin{aligned}
& \bar{\sigma}(\eta) \sqrt{2(\bar{c}(2 \eta)+\eta)} s \leq \varphi_{T-\varepsilon^{2} s^{-2}}+Y_{s}^{\varepsilon}(\omega) \leq \bar{\sigma}(\eta) \eta M^{-1} \varepsilon^{-2} \\
& u^{\varepsilon}\left(\varepsilon^{2} s, \varphi_{T-\varepsilon^{2} s}+\varepsilon^{2} Y_{s}^{\varepsilon}(\omega)\right) \leq 4 \exp -\eta s \\
& r\left(u^{\varepsilon}\left(\varepsilon^{2} s, \varphi_{T-\varepsilon^{2} s} \varepsilon^{-2}+\varepsilon^{2} Y_{s}^{\varepsilon}(\omega)\right)\right) \leq r(4 \exp -\eta s)
\end{aligned}
$$

and $r \in C^{1}[0,1]$ or $(2.10)$ yields dominated convergence in $s$.

\subsection{Proof of proposition 6.2.I.}

We can define on the same sample space (see remark 6.1.) the diffusions

$Y_{t}^{\varepsilon}=y+\int_{0}^{t} \sigma\left(\varphi_{T-\varepsilon^{2} s}+\varepsilon^{2} Y_{s}^{\varepsilon}\right) d W_{s}, \quad Y_{t}^{\prime} \varepsilon=y^{\prime}+\int_{0}^{t} \sigma\left(\varphi_{T-\varepsilon^{2} s}+\varepsilon^{2} Y_{s}^{\prime} \varepsilon\right) d W_{s}$.

Define $B_{\varepsilon}^{\prime}$ and $I_{\varepsilon}^{\prime}$ from $Y^{\prime} \varepsilon$ as $B_{\varepsilon}$ and $I_{\varepsilon}$ were defined from $Y^{\varepsilon}$ (see (6.3)).

Since $|y| \leq \varepsilon^{-\eta},(8.7)$ yields

$$
\begin{gathered}
P\left(\Omega \backslash B_{\varepsilon}\right), P\left(\Omega \backslash B_{\varepsilon}^{\prime}\right) \leq 2 \exp -\frac{\left(\varepsilon^{-1-\alpha}+\left|\varepsilon^{-\eta}\right|\right)^{2}}{2 \varepsilon^{a-2} M^{2}} \leq \exp -c s t \varepsilon^{-2 \alpha-a} \\
\begin{aligned}
g^{\varepsilon}(y)-g^{\varepsilon}\left(y^{\prime}\right) & =E\left[{ }^{1} B_{\varepsilon} \exp \left(-I_{\varepsilon}\right)-{ }^{1} B_{\varepsilon}^{\prime} \exp \left(-I_{\varepsilon}^{\prime}\right)\right] \\
& =E{ }^{1} B_{\varepsilon} \cap B_{\varepsilon}^{\prime}\left[\exp \left(-I_{\varepsilon}\right)-\exp \left(-I_{\varepsilon}^{\prime}\right)\right]+o(1) .
\end{aligned}
\end{gathered}
$$

Lemma 6.6. Let $\mu \in] 0, \inf (b, 3 a / 2-1)[$. Then

$$
P\left(\left\{\left\|Y^{\varepsilon}-Y^{\prime}\right\|_{0}^{\varepsilon^{a-2}} \geq \varepsilon^{\mu}\right\} \cap B_{\varepsilon} \cap B_{\varepsilon}^{\prime}\right)=o(1) .
$$


Proof. Let $X_{t}=y+\sigma(0) W_{t}, X_{t}^{\prime}=y^{\prime}+\sigma(0) W_{t}$ and $\left.\mu^{\prime} \in\right] \mu, \inf (b, 3 a / 2-1)[$. We get

$$
\begin{aligned}
P\left(\left\{\left\|Y^{\varepsilon}-Y^{\prime}\right\|_{0}^{\varepsilon^{a-2}} \geq \varepsilon^{\mu}\right\} \cap B_{\varepsilon} \cap B_{\varepsilon}^{\prime}\right) & \leq P\left(\left\{\left\|Y^{\varepsilon}-X\right\|_{0}^{\varepsilon^{a-2}} \geq \varepsilon^{\mu^{\prime}}\right\} \cap B_{\varepsilon}\right) \\
& +P\left(\left\{\left\|Y^{\varepsilon}-X^{\prime}\right\|_{0}^{\varepsilon^{a-2}} \geq \varepsilon^{\mu^{\prime}}\right\} \cap B_{\varepsilon}^{\prime}\right) .
\end{aligned}
$$

We have $Y_{t}^{\varepsilon}-X_{t}=\int_{0}^{t} A_{s}^{\varepsilon} d W_{s}$ where $A_{s}^{\varepsilon}=\sigma\left(\varphi_{T-\varepsilon^{2} s}+\varepsilon^{2} Y_{s}^{\varepsilon}\right)-\sigma(0)=O\left(\varepsilon^{a}\right)$ on $B_{\varepsilon}$ and if $s \leq \varepsilon^{a-2}$. Hence, (8.7) yields

$$
P\left(\left\{\left\|Y^{\varepsilon}-X\right\|_{0}^{\varepsilon^{a-2}} \geq \varepsilon^{\mu^{\prime}}\right\} \cap B_{\varepsilon}\right) \leq \exp -\operatorname{cst} \varepsilon^{2+2 \mu^{\prime}-3 a} .
$$

Let $D_{\varepsilon}=\left\{\left\|Y^{\varepsilon}-Y^{\prime \varepsilon}\right\|_{0}^{\varepsilon^{a-2}} \leq \varepsilon^{\mu}\right\} \cap B_{\varepsilon} \cap B_{\varepsilon}^{\prime}$. Lemma 6.4 yields

$$
\left|g^{\varepsilon}(y)-g^{\varepsilon}\left(y^{\prime}\right)\right| \leq E 1_{D_{\varepsilon}}\left|I_{\varepsilon}-I_{\varepsilon}^{\prime}\right|+o(1) .
$$

On the event $D_{\varepsilon}$ and if $s \leq \varepsilon^{a-2}$, then $\varphi_{T-\varepsilon^{2} s}+\varepsilon^{2} Y_{s}^{\varepsilon}$ and $\varphi_{T-\varepsilon^{2} s}+\varepsilon^{2} Y_{s}^{\prime} \varepsilon$ stay in a compact $K$. Let $k_{1}=\sup _{K}|c|$ and $k_{2}=\sup _{K}\left|c^{\prime}\right|$. We get

$$
\begin{aligned}
& { }^{1} D_{\varepsilon}\left|I_{\varepsilon}-I_{\varepsilon}^{\prime}\right| \leq k_{2} \varepsilon^{a-2} \varepsilon^{2+\mu} \\
& \quad+k_{1} \int_{0}^{\varepsilon^{a-2}}\left|r\left(u^{\varepsilon}\left(\varepsilon^{2} s, \varphi_{T-\varepsilon^{2} s}+\varepsilon^{2} Y_{s}^{\varepsilon}\right)\right)-r\left(u^{\varepsilon}\left(\varepsilon^{2} s, \varphi_{T-\varepsilon^{2} s}+\varepsilon^{2} Y_{s}^{\prime} \varepsilon\right)\right)\right| d s .
\end{aligned}
$$

Therefore $\left|g^{\varepsilon}(y)-g^{\varepsilon}\left(y^{\prime}\right)\right| \leq k_{1} l^{\varepsilon}\left(0, \varepsilon^{a-2}\right)+o(1)$ where $l^{\varepsilon}\left(a_{1}, a_{2}\right)=$

$$
E 1 D_{\varepsilon} \int_{a_{1}}^{a_{2}}\left|r\left(u^{\varepsilon}\left(\varepsilon^{2} s, \varphi_{T-\varepsilon^{2} s}+\varepsilon^{2} Y_{s}^{\varepsilon}\right)\right)-r\left(u^{\varepsilon}\left(\varepsilon^{2} s, \varphi_{T-\varepsilon^{2} s}+\varepsilon^{2} Y_{s}^{\prime} \varepsilon\right)\right)\right| d s .
$$

LEMMA 6.7. If $0<\gamma_{1}<\gamma_{2}<2 \gamma_{1} \leq 2-a$, then $l^{\varepsilon}\left(\varepsilon^{-\gamma_{1}}, \varepsilon^{-\gamma_{2}}\right)=o(1)$, where $o(1)$ depends only on $\gamma_{1}$ and $\gamma_{2}$, not on $y, y^{\prime}$.

Proof. According to (H4), there exists $\delta>0$ such that

$$
l=-\dot{\varphi}_{T}-\bar{\sigma}(\delta) \sqrt{2(\bar{c}(2 \delta)+\delta)}-\delta>0 .
$$

Let $V_{\varepsilon}^{1}=\left\{\varphi_{T-\varepsilon^{2} s} \varepsilon^{-2}+Y_{s}^{\varepsilon}<\bar{\sigma}(\delta) \delta M^{-1} \varepsilon^{-2}\right.$, for all $\left.s \in\left[\varepsilon^{-\gamma_{1}}, \varepsilon^{-\gamma_{2}}\right]\right\}$ and $V_{\varepsilon}^{2}=\{\bar{\sigma}(\delta) \sqrt{2(\bar{c}(2 \delta)+\delta}) s<\varphi_{T-\varepsilon^{2} s} \varepsilon^{-2}+Y_{s}^{\varepsilon}$, for all $\left.s \in\left[\varepsilon^{-\gamma_{1}}, \varepsilon^{-\gamma_{2}}\right]\right\}$.

$(8.7)$ yields that, for $\varepsilon$ small enough,

$$
\begin{aligned}
& P\left(\Omega \backslash V_{\varepsilon}^{1}\right) \leq P\left(\exists s \in\left[\varepsilon^{-\gamma_{1}}, \varepsilon^{-\gamma_{2}}\right] \quad Y_{s}^{\varepsilon}>c s t \varepsilon^{-2}\right) \leq \exp -c s t \varepsilon^{\gamma_{2}-4} \\
& P\left(\Omega \backslash V_{\varepsilon}^{2}\right) \leq P\left(\exists s \in\left[\varepsilon^{-\gamma_{1}}, \varepsilon^{-\gamma_{2}}\right] \quad Y_{s}^{\varepsilon}<-l s\right) \leq \exp -c s t \varepsilon^{\gamma_{2}-2 \gamma_{1}} .
\end{aligned}
$$

Let $C_{\varepsilon}=\int_{\varepsilon^{-\gamma_{1}}}^{\varepsilon^{-\gamma_{2}}} r\left(u^{\varepsilon}\left(\varepsilon^{2} s, \varphi_{T-\varepsilon^{2} s}+\varepsilon^{2} Y_{s}^{\varepsilon}\right)\right) d s$. Therefore, lemma 4.1 implies

$$
E C_{\varepsilon}=E 1^{1} V_{\varepsilon}^{1} \cap V_{\varepsilon}^{2} C_{\varepsilon}+o(1) \leq \int_{\varepsilon}^{\varepsilon^{-\gamma_{1}}} r(4 \exp -\delta s) d s+o(1)=o(1)
$$


The same computation for $Y^{\prime} \varepsilon$ ends the proof of lemma 6.5 . $o(1)$.

Now, let $\gamma \in] 0, \mu / 2\left[\right.$. By iterating lemma 6.5 , we get $l^{\varepsilon}\left(\varepsilon^{-\gamma}, \varepsilon^{a-2}\right)=$

It remains to prove $l^{\varepsilon}\left(0, \varepsilon^{-\gamma}\right)=o(1)$. Let $\left.\tau \in\right] \gamma / 2, \mu-3 \gamma / 2[$.

(8.7) yields

$$
P\left(\left\|Y^{\varepsilon}\right\|_{0}^{\varepsilon^{-\gamma}} \geq \varepsilon^{-\tau}\right) \leq \exp -\operatorname{cst} \varepsilon^{-2 \tau+\gamma} .
$$

Define $k^{\varepsilon}\left(a_{1}, a_{2}\right)$ by replacing $D_{\varepsilon}$ by

$$
\left.\left.\left\{\left\|Y^{\varepsilon}\right\|_{0}^{\varepsilon^{-\gamma}} \leq \varepsilon^{-\tau}\right)\right\} \cap\left\{\left\|Y^{\prime} \varepsilon\right\|_{0}^{\varepsilon^{-\gamma}} \leq \varepsilon^{-\tau}\right)\right\} \cap\left\{\left\|Y^{\varepsilon}-Y^{\varepsilon}\right\|_{0}^{\varepsilon^{-\gamma}} \leq \varepsilon^{\mu}\right\} \cap B_{\varepsilon} \cap B_{\varepsilon}^{\prime}
$$

in the definition of $l^{\varepsilon}\left(a_{1}, a_{2}\right)$. (6.8) yields $l^{\varepsilon}\left(0, \varepsilon^{-\gamma}\right)=k^{\varepsilon}\left(0, \varepsilon^{-\gamma}\right)+o(1)$.

Appendix 8.1 and $r \in C^{1}[0,1]$ imply

$$
\sup _{\varepsilon^{\gamma} \leq s \leq \varepsilon^{-\gamma}}\left|\partial_{x} r\left(u^{\varepsilon}\left(\varepsilon^{2} s, \varepsilon^{2} x\right)\right)\right|=O\left(\varepsilon^{-\gamma}\right)+O\left(\varepsilon^{-\gamma / 2}|x|\right) .
$$

Therefore,

$k^{\varepsilon}\left(0, \varepsilon^{-\gamma}\right)=k^{\varepsilon}\left(0, \varepsilon^{\gamma}\right)+k^{\varepsilon}\left(\varepsilon^{\gamma}, \varepsilon^{-\gamma}\right)=O\left(\varepsilon^{\gamma}\right)+O\left(\varepsilon^{\mu-\gamma}\left(\varepsilon^{-\gamma}+\varepsilon^{-\gamma / 2} \varepsilon^{-\tau}\right)\right)=o(1)$.

\subsection{Proof of proposition 6.1.iI}

Together with (6.2), we will use an explicit formula for $q^{\varepsilon}$, the transition density of $Y^{\varepsilon}$. Let

$$
\begin{aligned}
\gamma(x) & =\sigma\left(\varepsilon^{2} x\right), \alpha_{t}=\varepsilon^{-2} \varphi_{T-\varepsilon^{2} t} \\
G(t, x) & =\int_{0}^{x} \frac{d u}{\gamma\left(\alpha_{t}+u\right)}, \quad L(t, .) \text { be the inverse function of } G(t, .) \\
C(t, x) & =\partial_{t} G(t, L(t, x))-\frac{1}{2} \gamma^{\prime}\left(\alpha_{t}+L(t, x)\right) \\
& =\alpha_{t}^{\prime}\left[\gamma\left(\alpha_{t}+L(t, x)\right)^{-1}-\gamma\left(\alpha_{t}\right)^{-1}\right]-\frac{1}{2} \gamma^{\prime}\left(\alpha_{t}+L(t, x)\right) \\
D(t, x) & =-\frac{1}{2} \partial_{x} C(t, x)-\frac{1}{2} C^{2}(t, x)-\int_{0}^{x} \partial_{t} C(t, u) d u \\
H(t, x) & =\int_{0}^{G(t, x)} C(t, u) d u \\
& =\int_{0}^{x}\left(\alpha_{t}^{\prime}\left[\gamma\left(\alpha_{t}+v\right)^{-1}-\gamma\left(\alpha_{t}\right)^{-1}\right]-\frac{1}{2} \gamma^{\prime}\left(\alpha_{t}+v\right)\right) \frac{d v}{\gamma\left(\alpha_{t}+v\right)} \\
V_{u}(a, b) & =(1-u) a+u b \\
J(s, t, x, y) & = \\
& \mathbb{E}\left[\exp (t-s) \int_{0}^{1} D\left(V_{u}(s, t), V_{u}(G(s, x), G(t, y))+\sqrt{t-s} \mathbb{B}_{u}\right) d u\right]
\end{aligned}
$$

where $\mathbb{B}$ is a standard brownian bridge defined on another probability space $\Omega^{\prime}$ and $\mathbb{E}[\ldots]$ refers to $\Omega^{\prime}$ (as well as $\mathbb{P}(\ldots)$ later on). 
LEMMA 6.8 .

$$
\begin{aligned}
q_{s, t}^{\varepsilon}(x, y) & =\frac{1}{\sqrt{2 \pi(t-s)}} \frac{J(s, t, x, y)}{\gamma\left(\alpha_{t}+y\right)} \\
& \exp \left[-\frac{(G(t, y)-G(s, x))^{2}}{2(t-s)}+H(t, y)-H(s, x)\right]
\end{aligned}
$$

Proof. Straightforward extension of lemma 1 p. 268, Dacunha-Castelle and Florens-Zmirou (1986).

We will need the following bounds.

LEMMA 6.9 .

i) $\exists C_{1}>0 \quad \forall t, x \quad|H(t, x)| \leq C_{1} \varepsilon^{2}\left(1+x^{2}\right)$

ii) $\exists C_{2}>0 \quad \forall t, x \quad D(t, x) \leq C_{2} \varepsilon^{2}(1+|x|)$

iii) $\forall \lambda>0 \exists C_{3}(\lambda)>0 \quad\left|\varepsilon x_{\varepsilon}\right| \leq \lambda, \varepsilon^{2} t_{\varepsilon} \leq \varepsilon^{d} \Rightarrow\left|D\left(t_{\varepsilon}, x_{\varepsilon}\right)\right| \leq C_{3}(\lambda) \varepsilon^{2}$.

Proof . i) We have $\left\|\gamma^{\prime}\right\|_{\infty}=O\left(\varepsilon^{2}\right), \quad\left\|\gamma^{\prime \prime}\right\|_{\infty}=O\left(\varepsilon^{4}\right)$, and $\left\|\alpha^{\prime}\right\|_{\infty}<\infty$. Hence

$$
|H(t, x)| \leq c s t \varepsilon^{2}|x|+c s t\left|\int_{0}^{x}\left[\gamma\left(\alpha_{t}+v\right)-\gamma\left(\alpha_{t}\right)\right] d v\right| \leq c s t \varepsilon^{2}\left(1+x^{2}\right) .
$$

ii) Straightforward.

iii) Let $\alpha_{0}=\frac{\dot{\varphi}_{T} \sigma^{\prime}(0)}{\sigma(0)}$. Easy computations yield the successive results:

$$
\begin{aligned}
& \varepsilon^{2} L\left(t_{\varepsilon}, x_{\varepsilon}\right)=O(\varepsilon), \varepsilon^{2} \alpha_{t_{\varepsilon}}=p \varepsilon^{2} t_{\varepsilon}+O\left(\varepsilon^{2 d}\right), L\left(t_{\varepsilon}, x_{\varepsilon}\right)=\sigma(0) x_{\varepsilon}\left[1+O\left(\varepsilon^{d}\right)\right] \\
& C\left(t_{\varepsilon}, x_{\varepsilon}\right)=\alpha_{0} \varepsilon^{2} x_{\varepsilon}+O\left(\varepsilon^{2 d}\right), \quad \partial_{x} C\left(t_{\varepsilon}, x_{\varepsilon}\right)=\alpha_{0} \varepsilon^{2}+O\left(\varepsilon^{2+d}\right) \\
& \partial_{t} C\left(t_{\varepsilon}, x_{\varepsilon}\right)=c s t \varepsilon^{4} x_{\varepsilon}+O\left(\varepsilon^{2+2 d}\right), \quad D\left(t_{\varepsilon}, x_{\varepsilon}\right)=O\left(\varepsilon^{2}\right)
\end{aligned}
$$

According to $(6.2),(6.3)$ and lemma 6.6 , we have

$$
\begin{aligned}
& g^{\varepsilon}(y, z)-g^{\varepsilon}(y)=E 1_{B_{\varepsilon}} \exp \left(-I_{\varepsilon}\right)\left[J_{\varepsilon} \exp \left(G_{\varepsilon}+H_{\varepsilon}\right)-1\right] \\
& G_{\varepsilon}=\frac{\varepsilon^{2-d}}{2}\left[\left(G\left(\varepsilon^{d-2}, z \varepsilon^{-1}\right)-G(0, y)\right)^{2}\right. \\
& \left.-\left(1-\varepsilon^{a-d}\right)^{-1}\left(G\left(\varepsilon^{2-d}, z \varepsilon^{-1}\right)-G\left(\varepsilon^{a-2}, Y_{\varepsilon^{a-2}}^{\varepsilon}\right)\right)^{2}\right] \\
& H_{\varepsilon}=H(0, y)-H\left(\varepsilon^{a-2}, Y_{\varepsilon^{a}-2}^{\varepsilon}\right) \\
& J_{\varepsilon}=\frac{J_{1}^{\varepsilon}}{J_{2}^{\varepsilon}} \\
& J_{1}^{\varepsilon}=J\left(\varepsilon^{a-2}, \varepsilon^{d-2}, Y_{\varepsilon^{a-2}}^{\varepsilon}, z \varepsilon^{-1}\right) \\
& J_{2}^{\varepsilon}=J\left(0, \varepsilon^{d-2}, y, z \varepsilon^{-1}\right)
\end{aligned}
$$

As we expected, $J_{\varepsilon} \exp \left(G_{\varepsilon}+H_{\varepsilon}\right) \rightarrow 1$ a.s., and more precisely,

Lemma 6.10. For $\eta>0$ small enough, define $X_{\varepsilon}=\varepsilon^{\frac{1}{2}(2-a+\eta)}\left|Y_{\varepsilon^{a-2}}^{\varepsilon}\right|$.

i) $X_{\varepsilon} \rightarrow 0$ a.s. and $E \exp X_{\varepsilon}^{2} \rightarrow 1$

ii) $\left|G_{\varepsilon}\right|+\left|H_{\varepsilon}\right| \leq \alpha_{\varepsilon}\left(1+X_{\varepsilon}^{2}\right)$ a.s. where $\alpha_{\varepsilon} \rightarrow 0$. 
iii) $J_{1}^{\epsilon} \rightarrow 1$ a.s. and $J_{2}^{\epsilon} \rightarrow 1$.

The proof of this lemma is postponed to the end of the section.

In order to get a dominated convergence, we introduce

$$
\bar{B}_{\varepsilon}=B_{\varepsilon} \cap\left\{\left|Y_{\varepsilon^{a-2}}^{\varepsilon}\right| \leq \varepsilon^{-1}\right\}
$$

as well as $\bar{g}^{\varepsilon}(y)$ and $\bar{g}^{\varepsilon}(y, z)$ defined from $g^{\varepsilon}(y)$ and $g^{\varepsilon}(y, z)$ by replacing $B_{\varepsilon}$ by $\bar{B}_{\varepsilon}$.

It can be proved easily that $\bar{g}^{\varepsilon}(y)-g^{\varepsilon}(y) \rightarrow 0$.

Let us prove that $\bar{g}^{\complement}(y, z)-g^{\varepsilon}(y, z) \rightarrow 0$. According to lemma 6.7.ii,

$$
\begin{aligned}
J_{1}^{\varepsilon} & \leq \mathbb{E}\left[\exp \operatorname{cst} \varepsilon^{d} \int_{0}^{1}\left(1+|z| \varepsilon^{-1}+\left|Y_{\varepsilon^{a-2}}^{\varepsilon}\right|+\varepsilon^{d / 2-1}\left|\mathbb{B}_{u}\right|\right) d u\right] \text { a.s. } \\
& \leq \exp \left[\operatorname{cst} \varepsilon^{d-1}+\varepsilon^{d+a / 2-1-\eta} X_{\varepsilon}\right] \mathbb{E}\left[\exp \|\mathbb{B}\|_{0}^{1}\right] \text { a.s. }
\end{aligned}
$$

(notice that a.s. refers to the arguments of $J_{1}^{\varepsilon}, Y_{\varepsilon^{a-2}}^{\varepsilon}$ and $X_{\varepsilon}$ ).

Together with lemma 6.8 .ii and iii, it yields, for $\varepsilon$ small enough,

$$
\begin{aligned}
0 \leq g^{\varepsilon}(y, z)-\bar{g}^{\varepsilon}(y, z) & \leq\left(J_{2}^{\varepsilon}\right)^{-1} E 1_{\left\{\left|Y_{\varepsilon^{a-2}}^{\varepsilon}\right| \geq \varepsilon^{-1}\right\}} J_{1}^{\varepsilon} \exp \left[\alpha_{\varepsilon}\left(1+X_{\varepsilon}^{2}\right)\right] \\
& \leq \exp \operatorname{cst} \varepsilon^{d-1} E 1_{\left\{\left|Y_{\varepsilon^{a-2}}^{\varepsilon}\right| \geq \varepsilon^{-1}\right\}} \exp \beta_{\varepsilon}\left(1+X_{\varepsilon}^{2}\right)
\end{aligned}
$$

where $\beta_{\varepsilon} \rightarrow 0$. Moreover, $P\left(\left|Y_{\varepsilon^{a-2}}^{\varepsilon}\right| \geq \varepsilon^{-1}\right) \leq \exp -c s t \varepsilon^{-a}$. Hence,

$$
0 \leq g^{\varepsilon}(y, z)-\bar{g}^{\varepsilon}(y, z) \leq \exp \left(\operatorname{cst} \varepsilon^{d-1}-\operatorname{cst} \varepsilon^{-a}\right)\left[E\left(\exp 2 \beta_{\varepsilon} X_{\varepsilon}^{2}\right)\right]^{\frac{1}{2}}
$$

and we can conclude by using lemma 6.8 i and $a>1-d$.

It remains to prove that $\bar{g}^{\complement}(y, z)-\bar{g}^{\complement}(y) \rightarrow 0$. We introduced $\bar{B}_{\varepsilon}$ because

$$
{ }^{1}\left\{\left|Y_{\varepsilon^{a-2}}^{\varepsilon}\right| \leq \varepsilon^{-1}\right\} J_{1}^{\varepsilon} \leq c s t
$$

(actually, lemma 6.9.iii below is still valid when $z=O\left(\varepsilon^{1-\nu}\right)$ ). Therefore $\left|\bar{g}^{\varepsilon}(y, z)-\bar{g}^{\varepsilon}(y)\right| \leq\left|\bar{g}^{\varepsilon}(y, z)-E 1 \bar{B}_{\varepsilon} J_{\varepsilon} \exp \left(-I_{\varepsilon}\right)\right|+\left|\bar{g}^{\varepsilon}(y)-E 1 \bar{B}_{\varepsilon} J_{\varepsilon} \exp \left(-I_{\varepsilon}\right)\right|$.

The second term is smaller than $E 1_{\left\{\left|Y_{\varepsilon^{a-2}}^{c}\right| \leq \varepsilon^{-1}\right\}}\left|J_{\varepsilon}-1\right|$ which goes to 0 according to $(6.10)$.

By $(6.10)$, the first term is smaller than cst $E\left|\exp \left(G_{\varepsilon}+H_{\varepsilon}\right)-1\right|$ which goes to 0 by lemma 6.8.i and 6.8.ii.

Proof of lemma 6.8. i) The first part is a consequence of (8.8).

The second part holds since $\left(\exp X_{\varepsilon}^{2}\right)_{\varepsilon>0}$ is uniformly integrable. Actually

$$
\begin{aligned}
E \exp 2 X_{\varepsilon}^{2} & =\int_{0}^{+\infty} P\left(\exp 2 X_{\varepsilon}^{2} \geq y\right) d y=1+\int_{0}^{+\infty} P\left(X_{\varepsilon} \geq \sqrt{\frac{r}{2}}\right) \exp r d r \\
& \leq 1+\int_{0}^{1} \exp r d r+\int_{1}^{+\infty} \exp \left(r-c s t \frac{r}{\varepsilon^{\eta}}\right) d r \leq c s t \text { for } \varepsilon \text { small enough } .
\end{aligned}
$$


ii) Lemma 6.7.i and $|G(t, x)| \leq m^{-1}|x|$ yield

$$
\begin{aligned}
& \left|H\left(\varepsilon^{a-2}, Y^{\varepsilon}-\varepsilon^{a-2}\right)\right| \leq C_{1} \varepsilon^{2}\left(1+\varepsilon^{a-2-\eta} X_{\varepsilon}^{2}\right) \\
& 2 \varepsilon^{2-d}\left|G_{\varepsilon}\right| \leq \frac{\varepsilon^{a-d} z^{2} \varepsilon^{-2}}{1-\varepsilon^{a-d}}+c s t\left|z \varepsilon^{-1}\right|+\operatorname{cst}+\operatorname{cst}\left(Y_{\varepsilon^{\varepsilon-2}}^{\varepsilon}\right)^{2}+c s t\left|z \varepsilon^{-1} Y_{\varepsilon^{a-2}}^{\varepsilon}\right| \\
& \left|G_{\varepsilon}\right| \leq \operatorname{cst}\left[\varepsilon^{a-2 d+2-2 \nu}+o(1)+\varepsilon^{\frac{1}{2}(a-2 d-\eta)+1-\nu} X_{\varepsilon}+\varepsilon^{a-2 d-\eta} X_{\varepsilon}^{2}\right] .
\end{aligned}
$$

iii) In this proof, we restrict ourselves to the event $\left\{\varepsilon Y_{\varepsilon^{a-2}}^{\varepsilon} \rightarrow 0\right\}$ (it has probability 1 , see lemma 6.8.i). There exists $c$ such that $d>2 c>1-d$. Define

$$
\begin{aligned}
& \widetilde{J}_{1}^{e}=\mathbb{E}\left[1_{\left\{\|\mathbb{B}\|_{0}^{1} \leq \varepsilon^{-c}\right\}} \exp \left(\varepsilon^{d-2}-\varepsilon^{a-2}\right)\right. \\
& \left.\int_{0}^{1} D\left[V_{u}\left(\varepsilon^{a-2}, \varepsilon^{d-2}\right), V_{u}\left(G\left(\varepsilon^{a-2}, Y_{\varepsilon^{a}-2}^{\varepsilon}\right), G\left(\varepsilon^{d-2}, \frac{z}{\varepsilon}\right)\right)+\sqrt{\varepsilon^{d-2}-\varepsilon^{a-2}} \mathbb{B}_{u}\right] d u\right] .
\end{aligned}
$$

Lemma 6.7.ii yields that $\left|J_{1}-\widetilde{J}_{1}\right|$ is smaller than

$$
\begin{aligned}
\mathbb{E}\left[1_{\left\{\|\mathbb{B}\|_{0}^{1}\right.} \geq \varepsilon^{-c}\right\} & \left.\exp C_{2} \varepsilon^{d}\left[1+m^{-1}\left(Y_{\varepsilon^{a-2}}^{\varepsilon}+z \varepsilon^{-1}\right)+\varepsilon^{\frac{1}{2} d-1}\|\mathbb{B}\|_{0}^{1}\right]\right] \\
& \leq \exp \operatorname{cst} \varepsilon^{d-1} \mathbb{E}\left[1^{1}\left\{\|\mathbb{B}\|_{0}^{1} \geq \varepsilon^{-c}\right\}\right. \\
& \left.\leq \exp \|\mathbb{B}\|_{0}^{1}\right] \\
& \left.\operatorname{est} \varepsilon^{d-1}-\operatorname{cst} \varepsilon^{-2 c}\right)=o(1)
\end{aligned}
$$

We now prove that $\widetilde{J}_{1}^{e} \rightarrow 1$ a.s..

There exists $\lambda>0$ such that, a.s. on $\left\{\|\mathbb{B}\|_{0}^{1} \leq \varepsilon^{-c}\right\}$,

$\varepsilon\left|V_{u}\left(G\left(\varepsilon^{a-2}, Y_{\varepsilon^{a}-2}^{\varepsilon}\right), G\left(\varepsilon^{d-2}, z \varepsilon^{-1}\right)\right)+\sqrt{\varepsilon^{d-2}-\varepsilon^{a-2}} \mathbb{B}_{u}\right| \leq c s t+\varepsilon^{\frac{1}{2} d-c} \leq \lambda$.

Since $\varepsilon^{2} V_{u}\left(\varepsilon^{a-2}, \varepsilon^{d-2}\right) \leq \varepsilon^{d}$, lemma 6.7 .iii yields

$$
\left|\widetilde{J}_{1}^{c}-\mathbb{P}\left(|| \mathbb{B} \|_{0}^{1} \leq \varepsilon^{-c}\right)\right| \leq \exp \left(C_{3}(\lambda) \varepsilon^{d}\right)-1,
$$

which yields $\widetilde{J}_{1}^{\varepsilon} \rightarrow 1$ a.s.. We prove $J_{2}^{\varepsilon} \rightarrow 1$ in the same way.

\subsection{Proof of proposition 6.2.il.}

We will need the following results

Lemma 6.11. Define $X_{\varepsilon}=\varepsilon^{\frac{1}{2}(2-a+\eta)}\left|Y_{\varepsilon^{a-2}}^{\varepsilon}\right|$ as in lemma 6.8. Then

i) $\left(\exp X_{\varepsilon}^{2}\right)_{\varepsilon>0}$ is bounded in $L^{q}$, for all $q \geq 1$

ii) $G_{\varepsilon}+H_{\varepsilon} \leq \alpha_{\varepsilon}\left(1+X_{\varepsilon}^{2}\right) \quad$ where $\alpha_{\varepsilon} \rightarrow 0$

iii) $J_{1}^{\varepsilon} \leq \operatorname{cst}$ on $\left\{\left|Y_{\varepsilon^{a-2}}^{\varepsilon}\right| \leq \varepsilon^{-1}\right\}$

iv) $J_{2}^{\varepsilon} \geq \frac{1}{2} \quad$ for $\varepsilon$ small enough

v) $J_{1}^{\varepsilon} \leq$ cst $\exp X_{\varepsilon} \quad$ for $\varepsilon$ small enough.

All these results remain valid if we replace $X_{\varepsilon}, G_{\varepsilon}, \ldots$ by $X_{\varepsilon}^{\prime}, G_{\varepsilon}^{\prime}, \ldots$ 
Proof. i) and ii) The proofs are similar to that of lemmas 6.8.i and 6.8.ii. The fact that $y$ is not fixed but satisfies only $|y| \leq \varepsilon^{-\eta}$ does not alter the result. iii) There exists $k_{0}>0$ such that, on $\left\{\left|Y_{\varepsilon^{a-2}}^{c}\right| \leq \varepsilon^{-1}\right\}$, we have

$$
\mid V_{u}\left(G\left(\varepsilon^{a-2}, Y_{\varepsilon^{a-2}}^{\varepsilon}, G\left(\varepsilon^{d-2}, z \varepsilon^{-1}\right)\right) \mid \leq k_{0} \varepsilon^{-1}\right.
$$

According to lemma 6.7.ii and iii, on $\left\{\left|Y_{\varepsilon^{a-2}}^{\varepsilon}\right| \leq \varepsilon^{-1}\right\}$, we have

$$
\begin{aligned}
J_{1}^{\varepsilon} \leq \mathbb{P}\left(\|\mathbb{B}\|_{0}^{1} \leq \varepsilon^{-d / 2}\right) \exp C_{3}\left(k_{0}+1\right) \varepsilon^{d} \\
\quad+\mathbb{E}\left[1\left\{\|\mathbb{B}\|_{0}^{1} \geq \varepsilon^{-d / 2}\right\}\right. \\
\left.\quad \exp C_{2} \varepsilon^{d}\left(1+c s t \varepsilon^{-1}+\varepsilon^{d / 2-1}\|\mathbb{B}\|_{0}^{1}\right)\right] \\
=O\left(1+\exp \left(\text { cst } \varepsilon^{d-1}-c s t \varepsilon^{-d}\right)\right)=O(1)
\end{aligned}
$$

iv) We use lemma 6.7.iii to find a lower bound for $D$.

Since $|y|,\left|z \varepsilon^{-1}\right| \leq \varepsilon^{-1}$, on $\left\{\|\mathbb{B}\|_{0}^{1} \leq \varepsilon^{-d / 2}\right\}$,

$$
\left|V_{u}\left(G(0, y), G\left(\varepsilon^{d-2}, z \varepsilon^{-1}\right)\right)+\varepsilon^{d / 2-1} \mathbb{B}_{u}\right| \leq\left(m^{-1}+1\right) \varepsilon^{-1} .
$$

Therefore: $J_{2}^{\varepsilon} \geq \mathbb{P}\left(\|\mathbb{B}\|_{0}^{1} \leq \varepsilon^{-\frac{1}{2} d}\right) \exp \left[-C_{3}\left(m^{-1}+1\right) \varepsilon^{d}\right] \geq \frac{1}{2}$.

v) By lemma 6.7.ii,

$$
J_{1}^{\varepsilon} \leq \exp C_{2} \varepsilon^{d}\left(1+\operatorname{cst} Y_{\varepsilon^{a-2}}^{\varepsilon}+c s t|z| \varepsilon^{-1}\right) \leq \operatorname{cst} \exp X_{\varepsilon} .
$$

We now prove prop. 6.2.ii., ie $g^{\varepsilon}(y, z)-g^{\varepsilon}\left(y^{\prime}, z^{\prime}\right)=o(1)$.

As $B_{\varepsilon}, I_{\varepsilon}, G_{\varepsilon}, H_{\varepsilon}, J_{\varepsilon}, J_{1}^{\varepsilon}$ and $J_{2}^{\varepsilon}$ where defined from $Y^{\varepsilon}$ and $z$, we define $B_{\varepsilon}^{\prime}, I_{\varepsilon}^{\prime} \ldots$ from $Y^{\prime} \varepsilon$ and $z^{\prime}$ (see proof of prop. 6.2.i). Then,

$$
\begin{aligned}
& g^{\varepsilon}(y, z)-g^{\varepsilon}\left(y^{\prime}, z^{\prime}\right)= \\
& E\left[1_{B_{\varepsilon}} J_{\varepsilon} \exp \left(-I_{\varepsilon}+G_{\varepsilon}+H_{\varepsilon}\right)-{ }^{1} B_{\varepsilon}^{\prime} J_{\varepsilon}^{\prime} \exp \left(-I_{\varepsilon}^{\prime}+G_{\varepsilon}^{\prime}+H_{\varepsilon}^{\prime}\right)\right] .
\end{aligned}
$$

We define $k^{\varepsilon}\left(y, y^{\prime}, z, z^{\prime}\right)$ by replacing $B_{\varepsilon}$ and $B_{\varepsilon}^{\prime}$ by $B_{\varepsilon} \cap B_{\varepsilon}^{\prime}$ in the right-hand side member of $(6.11)$. Then,

$$
\begin{aligned}
\left|g^{\varepsilon}(y, z)-g^{\varepsilon}\left(y^{\prime}, z^{\prime}\right)-k^{\varepsilon}\left(y, y^{\prime}, z, z^{\prime}\right)\right| \leq & {\left[P\left(\Omega \backslash B_{\varepsilon}^{\prime}\right) E 1_{B_{\varepsilon}} J_{\varepsilon}^{2} \exp 2\left(G_{\varepsilon}+H_{\varepsilon}\right)\right]^{\frac{1}{2}} } \\
& +\left[P\left(\Omega \backslash B_{\varepsilon}\right) E 1_{B_{\varepsilon}^{\prime}}^{\prime} J_{\varepsilon}^{2} \exp 2\left(G_{\varepsilon}^{\prime}+H_{\varepsilon}^{\prime}\right)\right]^{\frac{1}{2}}
\end{aligned}
$$

(8.7) yields $P\left(\Omega \backslash B_{\varepsilon}^{\prime}\right)=o(1)$ and $P\left(\Omega \backslash B_{\varepsilon}\right)=o(1)$. Lemma 6.9.i, ii, iv and v, yield that $E 1_{B_{\varepsilon}} K_{\varepsilon}^{2} \exp 2\left(G_{\varepsilon}+H_{\varepsilon}\right)$ and $E 1_{B_{\varepsilon}^{\prime}} K_{\varepsilon}^{\prime 2} \exp 2\left(G_{\varepsilon}^{\prime}+H_{\varepsilon}^{\prime}\right)$ are bounded. Therefore, $g^{\varepsilon}(y, z)-g^{\varepsilon}\left(y^{\prime}, z^{\prime}\right)=k^{\varepsilon}\left(y, y^{\prime}, z, z^{\prime}\right)+o(1)$.

We now fix $\mu$ in $] 0, \inf (b, 3 a / 2-1)\left[\right.$ and define $g^{\varepsilon}\left(y, y^{\prime}, z, z^{\prime}\right)$ by replacing $B_{\varepsilon}$ and $B_{\varepsilon}^{\prime}$ by $B_{\varepsilon}^{\prime \prime}$ in $(6.11)$, where

$B_{\varepsilon}^{\prime \prime}=\left\{\| Y^{\varepsilon}-\left.Y^{\prime}\right|_{0} ^{\varepsilon^{a-2}} \leq \varepsilon^{\mu}\right\} \cap\left\{\left|Y_{\varepsilon^{a-2}}^{\varepsilon}\right| \leq \varepsilon^{-1}\right\} \cap\left\{\left|Y_{\varepsilon^{a-2}}^{\prime}\right| \leq \varepsilon^{-1}\right\} \cap B_{\varepsilon} \cap B_{\varepsilon}^{\prime}$. 
Lemma 6.4 yields $P\left(B_{\varepsilon} \cap B_{\varepsilon}^{\prime} \cap\left(\Omega \backslash B_{\varepsilon}^{\prime \prime}\right)\right)=o(1)$, and the same computation as before thus implies $k^{\varepsilon}\left(y, y^{\prime}, z, z^{\prime}\right)=g^{\varepsilon}\left(y, y^{\prime}, z, z^{\prime}\right)+o(1)$.

It is therefore enough to prove that $g^{\varepsilon}\left(y, y^{\prime}, z, z^{\prime}\right)=o(1)$. Since $a_{1} \ldots a_{n}-a_{1}^{\prime} \ldots a_{n}^{\prime}=\sum_{j=1}^{n}\left(\prod_{i=1}^{j-1} a_{i}^{\prime}\right)\left(a_{j}-a_{j}^{\prime}\right)\left(\prod_{i=j+1}^{n} a_{i}\right)$, lemma 6.9.iii and iv yield $\left|g^{\varepsilon}\left(y, y^{\prime}, z, z^{\prime}\right)\right| \leq \operatorname{cst}\left(g_{1}^{\varepsilon}+g_{2}^{\varepsilon}+g_{3}^{\varepsilon}+g_{4}^{\varepsilon}\right)$ where

$$
\begin{aligned}
& g_{1}^{\varepsilon}=E 1_{B_{\varepsilon}^{\prime \prime}}\left|I_{\varepsilon}-I_{\varepsilon}^{\prime}\right| \exp \left(G_{\varepsilon}+H_{\varepsilon}\right), \quad g_{2}^{\varepsilon}=E 1_{B_{\varepsilon}^{\prime \prime}}\left|\exp G_{\varepsilon}-\exp G_{\varepsilon}^{\prime}\right| \exp H_{\varepsilon} \\
& g_{3}^{\varepsilon}=E 1_{B_{\varepsilon}^{\prime \prime}}\left|\exp H_{\varepsilon}-\exp H_{\varepsilon}^{\prime}\right| \exp G_{\varepsilon}^{\prime}, \quad g_{4}^{\varepsilon}=E 1_{B_{\varepsilon}^{\prime \prime}}^{\prime \prime} J_{\varepsilon}-J_{\varepsilon}^{\prime} \mid \exp \left(G_{\varepsilon}^{\prime}+H_{\varepsilon}^{\prime}\right) .
\end{aligned}
$$

We conclude by proving that, for $i=1$ to $4, g_{i}^{\varepsilon}=o(1)$.

$\mathrm{i}=1$ : We change slightly the proof of $E 1_{D_{\varepsilon}}\left|I_{\varepsilon}-I_{\varepsilon}^{\prime}\right|=o(1)$ (proof of prop. $6.2 . \mathrm{i})$ by using lemma $6.9 . \mathrm{i}$ and ii.

$\mathrm{i}=2: \quad\left|(a-b)^{2}-\left(a^{\prime}-b^{\prime}\right)^{2}\right| \leq 4 \sup \left(|a|,\left|a^{\prime}\right|,|b|,\left|b^{\prime}\right|\right)\left(\left|a-a^{\prime}\right|+\left|b-b^{\prime}\right|\right)$ yields

$$
\left[G\left(\varepsilon^{d-2}, z \varepsilon^{-1}\right)-G(0, y)\right]^{2}-\left[G\left(\varepsilon^{d-2}, z^{\prime} \varepsilon^{-1}\right)-G\left(0, y^{\prime}\right)\right]^{2}=O\left(\varepsilon^{\frac{3}{2} d-2}\right) .
$$

In the same way, on $B_{\varepsilon}^{\prime \prime}$,

$$
\begin{array}{r}
{\left[G\left(\varepsilon^{d-2}, z \varepsilon^{-1}\right)-G\left(\varepsilon^{a-2}, Y_{\varepsilon^{a-2}}^{\varepsilon}\right)\right]^{2}-\left[G\left(\varepsilon^{d-2}, z^{\prime} \varepsilon^{-1}\right)-G\left(\varepsilon^{a-2}, Y_{\varepsilon^{a}-2}^{\prime}\right)\right]^{2}} \\
=O\left(\varepsilon^{d+\eta-2}\right) .
\end{array}
$$

Therefore ${ }^{\prime \prime} B_{\varepsilon}^{\prime \prime}\left|G_{\varepsilon}-G_{\varepsilon}^{\prime}\right| \leq \varepsilon^{\mu^{\prime}}$ where $\mu^{\prime}>0$. Hence

$$
g_{2}^{\varepsilon}=E\left[1_{B_{\varepsilon}^{\prime \prime}}\left|\exp \left(G_{\varepsilon}^{\prime}-G_{\varepsilon}\right)-1\right| \exp \left(G_{\varepsilon}+H_{\varepsilon}\right)\right] \leq \operatorname{cst}\left(\exp \varepsilon^{\mu^{\prime}}-1\right) .
$$

$\mathrm{i}=3$ : Since

$$
\left|H(t, x)-H\left(t, x^{\prime}\right)\right| \leq\|C\|_{\infty}\left|G(t, x)-G\left(t, x^{\prime}\right)\right| \leq m^{-1}\|C\|_{\infty}\left|x-x^{\prime}\right|,
$$

we have ${ }^{B_{\varepsilon}^{\prime \prime}}\left|H_{\varepsilon}-H_{\varepsilon}^{\prime}\right|=O\left(\varepsilon^{\mu}\right)$ and thus $g_{3}^{\varepsilon}=O\left(\exp \left(\right.\right.$ cst $\left.\left.\varepsilon^{\mu}\right)-1\right)$.

$\mathrm{i}=4$ : Inequality $\left|D(t, x)-D\left(t, x^{\prime}\right)\right| \leq c s t \varepsilon^{2}\left|x-x^{\prime}\right|$ yields

$$
\begin{gathered}
\left|J_{1}^{\varepsilon}-J_{1}^{\prime} \varepsilon\right| \leq J_{1}^{\varepsilon}\left[\exp \left(\operatorname{cst} \varepsilon^{d} \int_{0}^{1} A_{u}^{\varepsilon} d u\right)-1\right] \text { where } A_{u}^{\varepsilon} \text { equals } \\
\left|V_{u}\left(G\left(\varepsilon^{a-2}, Y_{\varepsilon^{a-2}}^{\varepsilon}\right), G\left(\varepsilon^{d-2}, z \varepsilon^{-1}\right)\right)-V_{u}\left(G\left(\varepsilon^{a-2}, Y_{\varepsilon^{\prime} \varepsilon-2}^{a}\right), G\left(\varepsilon^{d-2}, z^{\prime} \varepsilon^{-1}\right)\right)\right| .
\end{gathered}
$$

Let $k(\varepsilon)=\exp \left(c s t \varepsilon^{2 d+\eta-1}\right)-1$. Then

$$
{ }^{1} B_{\varepsilon}^{\prime \prime} A_{u}^{\varepsilon} \leq m^{-1}\left(\varepsilon^{\mu}+\varepsilon^{d+\eta-1}\right) \Rightarrow{ }^{1} B_{\varepsilon}^{\prime \prime}\left|J_{1}^{\varepsilon}-J_{1}^{\prime} \varepsilon\right| \leq k(\varepsilon) J_{1}^{\varepsilon} .
$$

Similarly, $\left|J_{2}^{\varepsilon}-J_{2}^{\prime}{ }^{\epsilon}\right| \leq k(\varepsilon) J_{2}^{\varepsilon}$ and $g_{4}^{\varepsilon} \leq \operatorname{cst} k(\varepsilon) E \exp \left(G_{\varepsilon}^{\prime}+H_{\varepsilon}^{\prime}\right)=o(1)$. 


\section{END OF THE PROOF}

\subsection{The linear problem}

By lemma 3.1, we have to study

$$
v_{1}^{\varepsilon}=E 1_{\left\{Z_{T}^{\varepsilon} \leq 0,\left\|Z^{\varepsilon}\right\| \leq \rho\right\}} \exp \left[p \bar{Z}_{T}^{\varepsilon} \varepsilon^{-2}+K\left(g_{1}\right)\right] .
$$

Let $\gamma>0$ small enough. Lemma 5.1 yields the existence of $Q=$ $\left(Q_{0}, Q_{1}, K_{0}, K_{1}\right)$ independent of $Y=-g_{1}(T)$ and $Q_{2}, K_{2} \in \mathbb{R}$ such that

$$
\begin{aligned}
& \bar{Z}_{T}^{\varepsilon}=\varepsilon J_{\varepsilon}^{Q}(Y) \text { where } J_{\varepsilon}^{Q}(y)=-y+\varepsilon\left(Q_{0}+Q_{1} y+Q_{2} y^{2}\right) \\
& K\left(g_{1}\right)=K_{0}+K_{1} Y+K_{2} Y^{2} \text { and } K_{0}=K\left(g_{1}^{0}\right) \\
& P\left(\Omega \backslash D_{\varepsilon} Q\right)=O\left(\exp -\varepsilon^{-c s t}\right)
\end{aligned}
$$

where $D_{\varepsilon}\left(A_{1}, \ldots, A_{n}\right)=\left\{\left|A_{i}\right| \leq \varepsilon^{-\gamma}, i=1, \ldots, n\right\}$.

Lemma 7.1. We have $v_{1}^{c}=v_{2}^{\varepsilon}+o(\varepsilon)$ where

$$
v_{2}^{\varepsilon}=E 1_{\left\{D_{\varepsilon}(Y, Q),-\varepsilon^{2-\gamma} \leq \bar{Z}_{T}^{\varepsilon} \leq 0\right\}} \exp \left[p \varepsilon^{-2} \bar{Z}_{T}^{\varepsilon}+K\left(g_{1}\right)\right] .
$$

Proof. Let $\bar{D}_{\varepsilon}=D_{\varepsilon}\left(Y, Q,\left\|\Gamma^{\varepsilon}\right\|\right)$ and

$$
\begin{aligned}
& \left.v_{3}^{\varepsilon}=E 1_{\left\{\bar{D}_{\varepsilon}\right.}, Z_{T}^{\varepsilon} \leq 0\right\} \exp \left[p \varepsilon^{-2} \bar{Z}_{T}^{\varepsilon}+K\left(g_{1}\right)\right] \\
& \bar{v}_{3}^{\varepsilon}=E 1_{\left\{\bar{D}_{\varepsilon}, \bar{Z}_{T}^{\varepsilon} \leq 0\right\}} \exp \left[p \varepsilon^{-2} \bar{Z}_{T}^{\varepsilon}+K\left(g_{1}\right)\right] \text {. }
\end{aligned}
$$

Azencott ((1980-81), p. 270) proved that there exists $C>0$ such that

$$
\rho r \geq C \Longrightarrow P\left(\left\|Z^{\varepsilon}\right\| \leq \rho,\left\|\Gamma^{\varepsilon}\right\| \geq r\right) \leq \exp -C^{-1} r^{2 / 3} .
$$

(7.1) and $P\left(\left\|Z^{\varepsilon}\right\| \geq \rho\right)=O\left(\exp -\varepsilon^{-c s t}\right)$ yield $P\left(\Omega \backslash \bar{D}_{\varepsilon}\right)=O\left(\exp -\varepsilon^{-c s t}\right)$.

Therefore, (3.10) implies $v_{1}^{\varepsilon}=v_{3}^{\varepsilon}+O\left(\exp -\varepsilon^{-c s t}\right)$. Moreover

$$
\begin{aligned}
\left|v_{3}^{\varepsilon}-\bar{v}_{3}^{\varepsilon}\right| & \leq E 1 \bar{D}_{\varepsilon}\left|1_{\left\{Z_{T}^{\varepsilon} \leq 0\right\}}-1_{\left\{\bar{Z}_{T}^{\varepsilon} \leq 0\right\}}\right| \exp \left[p \varepsilon^{-2} \bar{Z}_{T}^{\varepsilon}+K\left(g_{1}\right)\right] \\
& \leq E 1_{\left\{D_{\varepsilon},\left|\bar{Z}_{T}^{\varepsilon}\right| \leq \varepsilon^{3-\gamma}\right\}} \exp \left[p \varepsilon^{1-\gamma}+K\left(g_{1}\right)\right] \\
& \leq \operatorname{cst} E 1_{\left\{D_{\varepsilon}(Y, Q),\left|J_{\varepsilon}^{Q}(Y)\right| \leq \varepsilon^{2-\gamma}\right\}} \exp \left(K_{0}+K_{1} Y+K_{2} Y^{2}\right) .
\end{aligned}
$$

Let $\xi=\int_{0}^{T} \sigma_{0}^{2}(s) d s$ and $K_{3}=K_{2}-\frac{1}{2 \xi}$. This last expression equals

$$
{ }^{E 1} D_{\varepsilon} Q H_{2}^{\varepsilon}(Q) \exp K_{0}
$$

where

$$
H_{2}^{\varepsilon}(Q)=\frac{1}{\sqrt{2 \pi \xi}} \int_{-\varepsilon^{-\gamma}}^{\varepsilon^{-\gamma}}\left\{\left|J_{\varepsilon}^{Q}(y)\right| \leq \varepsilon^{2-\gamma}\right\} \exp \left[K_{1} y+K_{3} y^{2}\right] d y .
$$


We need the following lemma whose proof is straightforward.

Lemma 7.2. On $D_{\varepsilon} Q$, the function $J_{\varepsilon}^{Q}$ is one-to-one from $\left[-\varepsilon^{-\gamma}, \varepsilon^{-\gamma}\right]$ to an interval $I_{\varepsilon}^{Q}$ which contains $[-1,1]$. Its inverse function $\Phi_{\varepsilon}^{Q}$ satisfies

$$
\forall u \in I_{\varepsilon}^{Q} \quad\left|\Phi_{\varepsilon}^{Q}(u)+u\right| \leq 3 \varepsilon^{1-3 \gamma} \text { and }\left|\frac{d \Phi_{\varepsilon}^{Q}}{d u}(u)+1\right| \leq 4 \varepsilon^{1-2 \gamma} .
$$

Therefore, on $D_{\varepsilon} Q$, we have

$$
H_{2}^{\epsilon}(Q)=-\frac{1}{\sqrt{2 \pi \xi}} \int_{I_{\varepsilon}^{Q}}^{1}\left\{|u| \leq \varepsilon^{2-\gamma}\right\} \exp \left[K_{1} \Phi_{\varepsilon}^{Q}(u)+K_{3} \Phi_{\varepsilon}^{Q}(u)^{2}\right] d \Phi_{\varepsilon}^{Q}(u) .
$$

Therefore, ${ }^{1} D_{\varepsilon} Q H_{2}^{\varepsilon}(Q)=O\left(\varepsilon^{2-\gamma}\right)$ and $v_{3}^{\varepsilon}=\bar{v}_{3}^{\varepsilon}+o(\varepsilon)$. Finally, the presence of $\exp p \varepsilon^{-2} \bar{Z}_{T}^{\varepsilon}$ and (3.10) yield $\bar{v}_{3}^{\varepsilon}=v_{2}^{\varepsilon}+O\left(\exp -\varepsilon^{-c s t}\right)$. where

Since $Q$ and $Y$ are independent, we have $v_{2}^{\varepsilon}=E 1_{D_{\varepsilon}} H_{1}^{\varepsilon}(Q) \exp K\left(g_{1}^{0}\right)$

$$
\begin{aligned}
& \sqrt{2 \pi \xi} H_{1}^{\varepsilon}(Q)=\int_{-\varepsilon^{-\gamma}}^{\varepsilon^{-\gamma}}\left\{-\varepsilon^{1-\gamma} \leq J_{\varepsilon}^{Q}(y) \leq 0\right\} \exp \left[\frac{p J_{\varepsilon}^{Q}(y)}{\varepsilon}+K_{1} y+K_{3} y^{2}\right] d y \\
& =-\int_{I_{\varepsilon}^{Q} \cap\left[-\varepsilon^{1-\gamma}, 0\right]} \exp \left[\frac{p u}{\varepsilon}+K_{1} \Phi_{\varepsilon}^{Q}(u)+K_{3} \Phi_{\varepsilon}^{Q}(u)^{2}\right] d \Phi_{\varepsilon}^{Q}(u) \\
& =-\varepsilon \int_{-\varepsilon^{-\gamma}}^{0} \exp \left[p v+K_{1} \Phi_{\varepsilon}^{Q}(\varepsilon v)+K_{3} \Phi_{\varepsilon}^{Q}(\varepsilon v)^{2}\right] \frac{d \Phi_{\varepsilon}^{Q}}{d u}(\varepsilon v) d v .
\end{aligned}
$$

Finally, joint dominated convergence in $\omega$ and $v$ ends the proof of th. 2.1:

$$
\frac{v_{2}^{\varepsilon}}{\varepsilon} \longrightarrow \frac{1}{\sqrt{2 \pi \xi}} \int_{-\infty}^{0} \exp p v d v E \exp K\left(g_{1}^{0}\right)
$$

\subsection{The nonlinear problem}

In this section, the proofs of the lemmas are postponed to the end.

By lemmas 3.2 and 4.2 , we have to study

$u_{2}^{\varepsilon}=E 1_{\left\{Z_{T}^{\varepsilon} \leq 0,\left\|Z^{\varepsilon}\right\| \leq \rho\right\}} \exp \left[p \varepsilon^{-2} \bar{Z}_{T}^{\varepsilon}+K\left(g_{1}\right)-\varepsilon^{-2} F_{\varepsilon}\left(T(\varepsilon), T, \varphi+Z^{\varepsilon}\right)\right]$.

In order to apply prop. 6.1 and 6.2 , we first condition with respect to $\sigma\left(Z_{s}^{\varepsilon}, s \leq T^{\prime}(\varepsilon) ; Z_{T}^{\varepsilon}\right)$ where $T^{\prime}(\varepsilon)=T-\varepsilon^{d}$ and $\left.d \in\right] 2 / 3, a[$. Let $\alpha \in] 0,1-a[$ and

$$
u_{3}^{\varepsilon}=E 1_{\left\{Z_{T}^{\varepsilon} \leq 0,\left\|Z^{\varepsilon}\right\| \leq \varepsilon^{1-\alpha}\right\}} \exp \left[p \varepsilon^{-2} \bar{Z}_{T}^{\varepsilon}+K\left(g_{1}\right)\right] g^{\varepsilon}\left(\varepsilon^{-2} Z_{T}^{\varepsilon}, \varepsilon^{-1} Z_{T^{\prime}(\varepsilon)}^{\varepsilon}\right) .
$$

The conditionning yields

LEMMA 7.3. $u_{2}^{\varepsilon}=u_{3}^{\varepsilon}+o(\varepsilon)$.

According to the strategy described in section 2.4, we introduce the following functional of $g_{1}$ and $g_{2}$

$$
\begin{aligned}
u_{4}^{\varepsilon}=E 1_{\left\{D_{\varepsilon}\left(V_{\varepsilon}, Q_{\varepsilon}\right),-\varepsilon^{2-\gamma} \leq \bar{Z}_{T}^{\varepsilon} \leq 0,\left|\bar{Z}_{T^{\prime}(\varepsilon)}^{\varepsilon}\right| \leq \varepsilon^{1+d / 2-\gamma}\right\}} \\
\quad \exp \left[p \varepsilon^{-2} \bar{Z}_{T}^{\varepsilon}+K\left(g_{1}\right)\right] g^{\varepsilon}\left(\varepsilon^{-2} \bar{Z}_{T}^{\varepsilon}, \varepsilon^{-1} \bar{Z}_{T^{\prime}(\varepsilon)}^{\varepsilon}\right) .
\end{aligned}
$$


where the r.v. $Q^{\varepsilon}$ is defined as follow.

By lemma 5.3, there exists $Q_{\varepsilon}=\left\{Q_{j k}^{\varepsilon}, R_{j k}^{\varepsilon}, K_{j k}^{\varepsilon}, 0 \leq j+k \leq 1\right\}$ independent of $V_{\varepsilon}=\left(Y, Y_{\varepsilon}\right)=\left(-g_{1}(T),-g_{1}\left(T^{\prime}(\varepsilon)\right)\right)$, and there exists $\left\{Q_{j k}^{\varepsilon}, R_{j k}^{\varepsilon}, K_{j k}^{\varepsilon}, j+k=2\right\} \in \mathbb{R}^{9}$ uniformly bounded in $\varepsilon$ such that:

$$
\begin{aligned}
& \bar{Z}_{T}^{\varepsilon}=\varepsilon M_{\varepsilon}^{Q}\left(Y, Y_{\varepsilon}\right) \text { where } M_{\varepsilon}^{Q}(y, z)=-y+\varepsilon \sum_{0 \leq j+k \leq 2} Q_{j k}^{\varepsilon} y^{k} z^{j} \\
& \bar{Z}_{T^{\prime}(\varepsilon)}^{\varepsilon}=\varepsilon N_{\varepsilon}^{Q}\left(Y, Y_{\varepsilon}\right) \text { where } N_{\varepsilon}^{Q}(y, z)=-z+\varepsilon \sum_{0 \leq j+k \leq 2} R_{j k}^{\varepsilon} y^{k} z^{j} \\
& K\left(g_{1}\right)=\sum_{0 \leq j+k \leq 2} K_{j k}^{\varepsilon} Y_{\varepsilon}^{j} Y^{k} \text { where } K_{00}^{\varepsilon}=K\left(G_{100}^{\varepsilon}\right) \\
& P\left(\Omega \backslash D_{\varepsilon} Q_{\varepsilon}\right)=O\left(\exp -\varepsilon^{-c s t}\right) .
\end{aligned}
$$

We now have to study $u_{4}^{\varepsilon}$ since

LEMMA 7.4. $u_{3}^{\varepsilon}=u_{4}^{\varepsilon}+o(\varepsilon)$.

Since $Q_{\varepsilon}$ and $V_{\varepsilon}$ are independent, $u_{4}^{\varepsilon}=E 1_{D_{\varepsilon} Q_{\varepsilon}} H_{3}^{\varepsilon}\left(Q_{\varepsilon}\right) \exp K_{00}^{\varepsilon}$ where

$$
\begin{gathered}
H_{3}^{\varepsilon}\left(Q_{\varepsilon}\right)=\mu_{\varepsilon} \iint_{\mathcal{C}\left(\varepsilon^{-\gamma}\right)}{ }^{1}\left\{-\varepsilon^{1-\gamma} \leq M_{\varepsilon}^{Q}(y, z) \leq 0,\left|N_{\varepsilon}^{Q}(y, z)\right| \leq \varepsilon^{d / 2-\gamma}\right\} \\
\quad \exp \left(p \varepsilon^{-1} M_{\varepsilon}^{Q}(y, z)+\sum_{1 \leq j+k \leq 2} K_{j k}^{\varepsilon} y^{k} z^{j}\right) g^{\varepsilon}\left(\varepsilon^{-1} M_{\varepsilon}^{Q}(y, z), N_{\varepsilon}^{Q}(y, z)\right) \\
\quad \exp \left(-\frac{z^{2}}{2 I_{0}^{T^{\prime}(\varepsilon)}}-\frac{(y-z)^{2}}{2 I_{T^{\prime}(\varepsilon)}^{T}}\right) d y d z \\
\mathcal{C}(r)=\{(y, z)|| y|,| z \mid \leq r\}, \xi_{s}^{t}=\int_{s}^{t} \sigma_{0}^{2}(u) d u, \mu_{\varepsilon}=(2 \pi)^{-1}\left[\xi_{0}^{T^{\prime}(\varepsilon)} \xi_{T^{\prime}(\varepsilon)}^{T}\right]^{-\frac{1}{2}}
\end{gathered}
$$

In order to compute this gaussian double integral, we use

Lemma 7.5. On $D_{\varepsilon} Q_{\varepsilon},\left(M_{\varepsilon}^{Q}, N_{\varepsilon}^{Q}\right)$ is a $C^{\infty}$-diffeomorphism from a neighborhood of $\mathcal{C}\left(\varepsilon^{-\gamma}\right)$ to a neighborhood of $\mathcal{C}(1)$. Let $\Lambda_{\varepsilon}^{Q}=\left(\Phi_{\varepsilon}^{Q}, \Psi_{\varepsilon}^{Q}\right)$ be its inverse function. Then, for all $(u, v) \in \mathcal{C}(1)$,

$$
\begin{aligned}
& \operatorname{Jac} \Lambda_{\varepsilon}^{Q}(u, v)=1+O\left(\varepsilon^{\gamma}\right) \text { and }\left|\Phi_{\varepsilon}^{Q}(u, v)+u\right|+\left|\Psi_{\varepsilon}^{Q}(u, v)+v\right|=O\left(\varepsilon^{2 \gamma}\right) . \\
& \text { Let } \lambda_{\varepsilon}=\sqrt{\xi_{T^{\prime}(\varepsilon)}^{T}} \text {. A change of variables yields (see lemma 7.5) }
\end{aligned}
$$

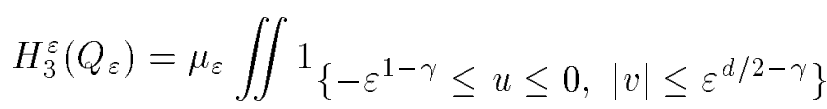

$$
\begin{aligned}
& \exp \left(\frac{p u}{\varepsilon}+\sum_{1 \leq j+k \leq 2} K_{j k}^{\varepsilon} \Phi_{\varepsilon}^{Q}(u, v)^{k} \Psi_{\varepsilon}^{Q}(u, v)^{j}\right) g^{\varepsilon}\left(\frac{u}{\varepsilon}, v\right) \\
& \exp \left(-\frac{\Psi_{\varepsilon}^{Q}(u, v)^{2}}{2 \xi_{0}^{T^{\prime}(\varepsilon)}}-\frac{\left(\Phi_{\varepsilon}^{Q}(u, v)-\Psi_{\varepsilon}^{Q}(u, v)\right)^{2}}{2 \xi_{T^{\prime}(\varepsilon)}^{T}}\right)\left|\operatorname{Jac} \Lambda_{\varepsilon}^{Q}(u, v)\right| d u d v
\end{aligned}
$$

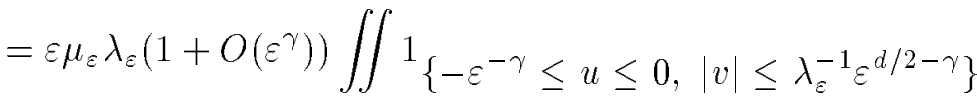

$$
\begin{aligned}
& \exp \left(p u+\sum_{1 \leq j+k \leq 2} K_{j k}^{\varepsilon} \Phi_{\varepsilon}^{Q}\left(\varepsilon u, \lambda_{\varepsilon} v\right)^{k} \Psi_{\varepsilon}^{Q}\left(\varepsilon u, \lambda_{\varepsilon} v\right)^{j}\right) g^{\varepsilon}\left(u, \lambda_{\varepsilon} v\right) \\
& \exp \left(-\frac{\Psi_{\varepsilon}^{Q}\left(\varepsilon u, \lambda_{\varepsilon} v\right)^{2}}{2 \xi_{0}^{T^{\prime}(\varepsilon)}}-\frac{\left(\Phi_{\varepsilon}^{Q}\left(\varepsilon u, \lambda_{\varepsilon} v\right)-\Psi_{\varepsilon}^{Q}\left(\varepsilon u, \lambda_{\varepsilon} v\right)\right)^{2}}{2 \xi_{T^{\prime}(\varepsilon)}^{T}}\right) d u d v
\end{aligned}
$$


The following lemma will allow us to end the proof of theorem 2.3.

Lemma 7.6. Let $D_{\varepsilon}^{\prime}=D_{\varepsilon}|| g_{1} \| \cap\left\{\left|Y-Y_{\varepsilon}\right| \leq \varepsilon^{d / 2-\gamma}\right\}$. Then,

i) $P\left(\Omega \backslash D_{\varepsilon}^{\prime}\right)=O\left(\exp -\varepsilon^{-c s t}\right)$ and ${ }^{1} D_{\varepsilon}^{\prime}\left|K_{00}^{\varepsilon}-K\left(g_{1}^{0}\right)\right|=O\left(\varepsilon^{\gamma}\right)$ a.s.

ii) There exists $\varepsilon_{0}>0$ such that $\left(\exp K_{00}^{\varepsilon}\right)_{\varepsilon \leq \varepsilon_{0}}$ is uniformly integrable.

iii) $\varepsilon^{-1} 1_{D_{\varepsilon}} Q_{\varepsilon} H_{3}^{\varepsilon}\left(Q_{\varepsilon}\right) \longrightarrow(2 \pi \xi)^{-\frac{1}{2}} \int_{-\infty}^{0} g(u) \exp p u d u$ a.s.

iv) There exists $M_{0}>0$ such that for all $\varepsilon, \varepsilon^{-1}{ }^{1} D_{\varepsilon} Q_{\varepsilon} H_{3}^{\varepsilon}\left(Q_{\varepsilon}\right) \leq M_{0}$ a.s.

Therefore,

$$
\begin{aligned}
\varepsilon^{-1} u_{4}^{\varepsilon} & =\varepsilon^{-1} E 1 D_{\varepsilon} Q_{\varepsilon} H_{3}^{\varepsilon}\left(Q_{\varepsilon}\right) \exp K_{00}^{\varepsilon} \\
& =\varepsilon^{-1} E 1 D_{\varepsilon}^{\prime} \cap D_{\varepsilon} Q_{\varepsilon} H_{3}^{\varepsilon}\left(Q_{\varepsilon}\right) \exp K_{00}^{\varepsilon}+O\left(\exp -\varepsilon^{-c s t}\right) \\
& =(2 \pi \xi)^{-\frac{1}{2}} \int_{-\infty}^{0} g(u) \exp p u d u E \exp K\left(g_{1}^{0}\right)+o(1) .
\end{aligned}
$$

Proof of lemma 7.3. (3.10) and (8.7) yield $u_{2}^{\varepsilon}=r_{1}^{\varepsilon}+o(\varepsilon)$ where

$$
\begin{aligned}
& r_{1}^{\varepsilon}=E 1_{A_{\varepsilon}} \exp \left[p \varepsilon^{-2} \bar{Z}_{T}^{\varepsilon}+K\left(g_{1}\right)-\varepsilon^{-2} F_{\varepsilon}\left(T(\varepsilon), T, \varphi+Z^{\varepsilon}\right)\right] \\
& A_{\varepsilon}=\left\{Z_{T}^{\varepsilon} \leq 0,\left\|Z^{\varepsilon}\right\| \leq \varepsilon^{1-\alpha}\right\} .
\end{aligned}
$$

Before conditioning with respect to $\mathcal{G}_{\varepsilon}=\sigma\left(Z_{s}^{\varepsilon}, s \leq T^{\prime}(\varepsilon) ; Z_{T}^{\varepsilon}\right)$, we introduce a $\mathcal{G}_{\varepsilon}$-measurable r.v. close to $p \varepsilon^{-2} \bar{Z}_{T}^{\varepsilon}+K\left(g_{1}\right)$. Integration by part in (2.6) yields

$$
K\left(g_{1}\right)=\int_{0}^{T} \psi_{1}(s) g_{1}^{2}(s) d s+\int_{0}^{T} \psi_{2}(s) g_{1}(s) d g_{1}(s)
$$

with continuous $\psi_{1}$ and $\psi_{2}$. Therefore, $K\left(g_{1}\right)=\kappa_{\varepsilon}+\kappa_{\varepsilon}^{\prime}$ where $\kappa_{\varepsilon}$ (resp. $\left.\kappa_{\varepsilon}^{\prime}\right)$ corresponds to the integrals on $\left[0, T^{\prime}(\varepsilon)\right]\left(\operatorname{resp} .\left[T^{\prime}(\varepsilon), T\right]\right)$. Hence

$$
p \varepsilon^{-2} \bar{Z}_{T}^{\varepsilon}+K\left(g_{1}\right)=p \varepsilon^{-2} Z_{T}^{\varepsilon}+\kappa_{\varepsilon}+U_{\varepsilon} \text { where } U_{\varepsilon}=-p \varepsilon \Gamma_{T}^{\varepsilon}+\kappa_{\varepsilon}^{\prime} .
$$

(7.1) yields that, for $\lambda \in]-\infty, \alpha[$,

$$
P\left(\left\|Z^{\varepsilon}\right\| \leq \varepsilon^{1-\alpha}, p \varepsilon\left|\Gamma_{T}^{\varepsilon}\right| \geq \varepsilon^{\lambda}\right) \leq \exp -\operatorname{cst} \varepsilon^{\frac{2}{3}(\lambda-1)} .
$$

Besides, for $\eta>0$ small enough, $P\left(\left|\kappa_{\varepsilon}^{\prime}\right| \geq 2 \varepsilon^{\lambda}\right)$ is smaller than

$$
\begin{gathered}
P\left(\left|\int_{T^{\prime}(\varepsilon)}^{T} \psi_{1}(s) g_{1}^{2}(s) d s\right| \geq \varepsilon^{\lambda}\right)+P\left(\left|\int_{T^{\prime}(\varepsilon)}^{T} \psi_{2}(s) g_{1}(s) \sigma_{0}(s) d W_{s}\right| \geq \varepsilon^{\lambda}\right) \\
\leq P\left(|| g_{1} \| \geq c s t \varepsilon^{\frac{1}{2}}(\lambda-d)\right)+P\left(|| g_{1} \| \geq \varepsilon^{-\eta}\right) \\
\quad+P\left(\left|\int_{T^{\prime}(\varepsilon)}^{T} \psi_{2}(s) g_{1}(s) \sigma_{0}(s) d W_{s}\right| \geq \varepsilon^{\lambda},\left\|g_{1}\right\| \leq \varepsilon^{-\eta}\right) \\
\leq \exp -c s t \varepsilon^{\lambda-d}+\exp -c s t \varepsilon^{-2 \eta}+\exp -c s t \varepsilon^{2 \lambda-d+2 \eta}
\end{gathered}
$$

$(\lambda<\alpha<1 / 3$ and $d>2 / 3)$. (7.2) and (7.3) with $\lambda \in] 0, \alpha[$ yield

$$
P\left(\left\|Z^{\varepsilon}\right\| \leq \varepsilon^{1-\alpha},\left|U_{\varepsilon}\right| \geq 3 \varepsilon^{\lambda}\right)=O\left(\exp -\varepsilon^{-c s t}\right) .
$$


(7.2) and (7.3) with $\lambda=-\eta$ yield the existence of $\eta^{\prime}>\eta$ such that

$$
P\left(\left\|Z^{\varepsilon}\right\| \leq \varepsilon^{1-\alpha},\left|U_{\varepsilon}\right| \geq 3 \varepsilon^{-\eta}\right)=O\left(\exp -\varepsilon^{-\eta^{\prime}}\right) .
$$

(7.5) imply that, for all $q \in \mathbb{R}$,

$$
\sup _{\varepsilon \leq \varepsilon_{0}} E 1_{\left\{\left\|Z^{\varepsilon}\right\| \leq \varepsilon^{1-\alpha}\right\}} \exp q\left|U_{\varepsilon}\right|<\infty .
$$

Let $r_{2}^{\varepsilon}=E 1_{A_{\varepsilon}} \exp \left[p \varepsilon^{-2} Z_{T}^{\varepsilon}+\kappa_{\varepsilon}-\varepsilon^{-2} F_{\varepsilon}\left(T(\varepsilon), T, \varphi+Z^{\varepsilon}\right)\right]$. Then,

$$
\left|r_{2}^{\varepsilon}-r_{1}^{\varepsilon}\right| \leq E 1_{A_{\varepsilon}} \exp \left[p \varepsilon^{-2} \bar{Z}_{T}^{\varepsilon}+K\left(g_{1}\right)\right]\left(\exp \left|U^{\varepsilon}\right|-1\right)
$$

Therefore, (3.10), (7.4) and (7.6) imply $r_{2}^{\varepsilon}-r_{1}^{\varepsilon}=o(\varepsilon)$.

With the same techniques, if we replace in $r_{2}^{\varepsilon}$ the event $A_{\varepsilon}$ by $\left\{Z_{T}^{\varepsilon} \leq\right.$ $\left.0,\left\|Z^{\varepsilon}\right\|_{0}^{T^{\prime}(\varepsilon)} \leq \varepsilon^{1-\alpha},\left\|Z^{\varepsilon}\right\|_{T(\varepsilon)}^{T} \leq \varepsilon^{1-\alpha}\right\}$, then the difference is $o(\varepsilon)$.

Finally, we condition on $\mathcal{G}_{\varepsilon}$ : the Markov property yields that $r_{2}^{\varepsilon}$ equals

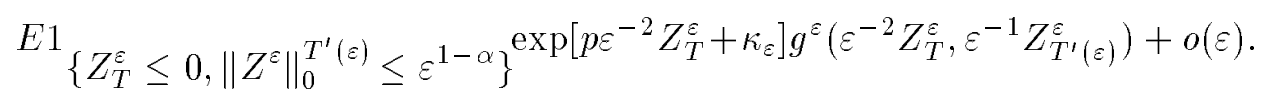

We conclude by proving $r_{2}^{\varepsilon}=u_{3}^{\varepsilon}+o(\varepsilon)$ (same techniques).

Proof of lemma 7.4. Let

$$
\begin{aligned}
& S_{\varepsilon}={ }^{1} D_{\varepsilon}\left(V_{\varepsilon}, Q_{\varepsilon},\left\|\Gamma^{\varepsilon}\right\|\right) \exp K\left(g_{1}\right) \\
& s_{1}^{\varepsilon}=E 1_{\left\{\left\|Z^{\varepsilon}\right\| \leq \varepsilon^{1-\alpha}, Z_{T}^{\varepsilon} \leq 0\right\}} S_{\varepsilon} \exp \left(p \varepsilon^{-2} \bar{Z}_{T}^{\varepsilon}\right) g^{\varepsilon}\left(\varepsilon^{-2} Z_{T}^{\varepsilon}, \varepsilon^{-1} Z_{T^{\prime}(\varepsilon)}^{\varepsilon}\right) \\
& s_{2}^{\varepsilon}=E 1_{\left\{\left\|Z^{\varepsilon}\right\| \leq \varepsilon^{1-\alpha}, \bar{Z}_{T}^{\varepsilon} \leq 0\right\}} S_{\varepsilon} \exp \left(p \varepsilon^{-2} \bar{Z}_{T}^{\varepsilon}\right) g^{\varepsilon}\left(\varepsilon^{-2} Z_{T}^{\varepsilon}, \varepsilon^{-1} Z_{T^{\prime}(\varepsilon)}^{\varepsilon}\right) .
\end{aligned}
$$

(3.10) and (7.1) yield $u_{3}^{\varepsilon}=s_{1}^{\varepsilon}+O\left(\exp -\varepsilon^{-c s t}\right)$. We have

$$
\begin{aligned}
& { }^{1} D_{\varepsilon}\left\|\Gamma^{\varepsilon}\right\|{ }^{1}\left\{Z_{T}^{\varepsilon} \leq 0\right\}-{ }^{-1}\left\{\bar{Z}_{T}^{\varepsilon} \leq 0\right\} \\
& \left.\left|s_{2}^{\varepsilon}-s_{1}^{\varepsilon}\right| \leq \operatorname{cst} E 1_{\left\{D_{\varepsilon} Y, \mid \exp p \varepsilon^{-2} \bar{Z}_{T}^{\varepsilon} \leq \exp p \varepsilon^{1-\gamma}\right.}{ }^{\varepsilon} \mid \varepsilon^{3-\gamma}\right\} \\
& \exp K\left(g_{1}\right) .
\end{aligned}
$$

We divide this last expectation into two parts by introducing ${ }^{1} D_{\varepsilon} Q$ and ${ }_{\Omega \backslash D_{\varepsilon} Q}$ (see section 7.1). The first part is $o(\varepsilon)$ (see proof of lemma 7.2), and the second one is $O\left(\exp -\varepsilon^{-c s t}\right)$. Hence $s_{1}^{\varepsilon}=s_{2}^{\varepsilon}+o(\varepsilon)$.

Write $s_{2}^{\varepsilon}=s_{3}^{\varepsilon}+s_{4}^{\varepsilon}$ where $s_{3}^{\varepsilon}$ (resp. $s_{4}^{\varepsilon}$ ) corresponds to ${ }\left\{-\varepsilon^{2-\gamma} \leq \bar{Z}_{T}^{\varepsilon}\right\}$ (resp. $1_{\left\{-\varepsilon^{2-\gamma}>\bar{Z}_{T}^{e}\right\}}$ ). Then $s_{4}^{\varepsilon}=O\left(\exp -\varepsilon^{-c s t}\right)$. Moreover, $(8.7)$ yields

$$
P\left(\left|Z_{T}^{\varepsilon}-Z_{T^{\prime}(\varepsilon)}^{\varepsilon}\right| \geq \varepsilon^{1+d / 2-\gamma}\right) \leq \exp -c s t \varepsilon^{-2 \gamma} .
$$

Since $\left\{D_{\varepsilon}\left\|\Gamma^{\varepsilon}\right\|,-\varepsilon^{2-\gamma} \leq \bar{Z}_{T}^{\varepsilon} \leq 0\right\} \subset\left\{\left|Z_{T}^{\varepsilon}\right| \leq 2 \varepsilon^{2-\gamma}\right\}$, inequality (7.7) yields

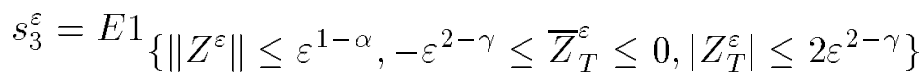

$$
\begin{aligned}
& { }^{1}\left\{\left|Z_{T^{\prime}(\varepsilon)}^{\varepsilon}\right| \leq 2 \varepsilon^{1+\frac{d}{2}-\gamma},\left|\bar{Z}_{T^{\prime}(\varepsilon)}^{\varepsilon}\right| \leq 3 \varepsilon^{1+d / 2-\gamma}\right\} \\
& S_{\varepsilon} \exp \left(p \varepsilon^{-2} \bar{Z}_{T}^{\varepsilon}\right) g^{\varepsilon}\left(\varepsilon^{-2} Z_{T}^{\varepsilon}, \varepsilon^{-1} Z_{T^{\prime}(\varepsilon)}^{\varepsilon}\right)+o(\varepsilon) \text {. }
\end{aligned}
$$


Define $s_{5}^{\varepsilon}$ by replacing $g^{\varepsilon}\left(\varepsilon^{-2} Z_{T}^{\varepsilon}, \varepsilon^{-1} Z_{T^{\prime}(\varepsilon)}^{\varepsilon}\right)$ by $g^{\varepsilon}\left(\varepsilon^{-2} \bar{Z}_{T}^{\varepsilon}, \varepsilon^{-1} \bar{Z}_{T^{\prime}(\varepsilon)}^{\varepsilon}\right)$ in the previous expectation. According to proposition 6.2.ii,

$$
\left|s_{3}^{\varepsilon}-s_{5}^{\varepsilon}\right| \leq o(1) E 1_{\left\{Z_{T}^{\varepsilon} \leq 0,\left\|Z^{\varepsilon}\right\| \leq \varepsilon^{1-\alpha}\right\}} \exp \left[p \varepsilon^{-2} \bar{Z}_{T}^{\varepsilon}+K\left(g_{1}\right)\right] .
$$

This last expectation is smaller than $v_{1}^{\varepsilon}$ if $\varepsilon^{1-\alpha} \leq \rho$. Therefore, theorem 2.1 yields $s_{3}^{\varepsilon}=s_{5}^{\varepsilon}+o(\varepsilon)$. Finally, easy computations imply $s_{5}^{\varepsilon}=u_{4}^{\varepsilon}+o(\varepsilon)$.

Proof of lemma 7.5. On $D_{\varepsilon} Q_{\varepsilon}$, for $(y, z) \in \mathcal{C}\left(\varepsilon^{-\gamma}\right)$,

$$
\begin{array}{ll}
\partial_{y} M_{\varepsilon}^{Q}(y, z)=-1+O\left(\varepsilon^{\gamma}\right) & \partial_{z} M_{\varepsilon}^{Q}(y, z)=O\left(\varepsilon^{\gamma}\right) \\
\partial_{y} N_{\varepsilon}^{Q}(y, z)=O\left(\varepsilon^{\gamma}\right) & \partial_{z} N_{\varepsilon}^{Q}(y, z)=-1+O\left(\varepsilon^{\gamma}\right) .
\end{array}
$$

Let $L_{\varepsilon}^{Q}=\left(M_{\varepsilon}^{Q}, N_{\varepsilon}^{Q}\right)$. Hence, Jac $L_{\varepsilon}^{Q}(y, z)=1+O\left(\varepsilon^{\gamma}\right)$. According to the local inversion theorem, there exists $V_{\varepsilon}^{Q}$, open neighborhood of $\mathcal{C}\left(\varepsilon^{-\gamma}\right)$, such that $L_{\varepsilon}^{Q}$ is a $C^{\infty}$-diffeomorphism from $V_{\varepsilon}^{Q}$ to $L_{\varepsilon}^{Q}\left(V_{\varepsilon}^{Q}\right)$. We can choose $V_{\varepsilon}^{Q}$ simply connected. Thus $L_{\varepsilon}^{Q}\left(V_{\varepsilon}^{Q}\right)$ is also simply connected. Consider a closed path whose support is the boundary of $\mathcal{C}\left(\varepsilon^{-\gamma}\right)$. Its image by $L_{\varepsilon}^{Q}$ is a closed path whose winding number with respect to $(0,0)$ is not zero and whose intersection with $\mathcal{C}(1)$ is empty (easy check). Hence $L_{\varepsilon}^{Q}\left(V_{\varepsilon}\right)$ contains $\mathcal{C}(1)$. The rest of the proof is straightforward.

Proof of lemma 7.6.i. The first part is a consequence of (8.7). According to $(5.2)$, we have

$$
\begin{aligned}
G_{100}^{\varepsilon}(t)-g_{1}^{0}(t) & =\left(\frac{\xi_{0}^{t}}{\xi_{0}^{T^{\prime}(\varepsilon)}}-\frac{\xi_{0}^{t}}{\xi_{0}^{T}}\right) Y_{\varepsilon}-\frac{\xi_{0}^{t}}{\xi_{0}^{T}}\left(Y-Y_{\varepsilon}\right) \quad \text { if } t \leq T^{\prime}(\varepsilon) \\
& =\left(1-\frac{\xi_{0}^{t}}{\xi_{0}^{T}}\right) Y_{\varepsilon}-\left(\frac{\xi_{0}^{t}}{\xi_{0}^{T}}-\frac{\xi_{T^{\prime}(\varepsilon)}^{t}}{\xi_{T^{\prime}(\varepsilon)}^{T}}\right)\left(Y-Y_{\varepsilon}\right) \quad \text { if } t \geq T^{\prime}(\varepsilon),
\end{aligned}
$$

and $\left\|G_{100}^{\varepsilon}-g_{1}^{0}\right\|=O\left(\varepsilon^{d}\left\|g_{1}\right\|+\left|Y-Y_{\varepsilon}\right|\right)=O\left(\varepsilon^{2 \gamma}\right)$ on $D_{\varepsilon}^{\prime}$.

(2.11) yields $K_{00}^{\varepsilon}-K\left(g_{1}^{0}\right)=O\left(\left\|\left(G_{100}^{c}\right)^{2}-\left(g_{1}^{0}\right)^{2}\right\|\right)$. Therefore,

$$
{ }^{1} D_{\varepsilon}^{\prime}\left|K_{00}^{\varepsilon}-K\left(g_{1}^{0}\right)\right|=O\left(\varepsilon^{2 \gamma}\left(\left\|G_{100}^{\varepsilon}+g_{1}^{0}\right\|\right)\right)
$$

and we conclude by using $\left\|G_{100}^{\varepsilon}\right\|+\left\|g_{1}^{0}\right\|=O\left(\left\|g_{1}\right\|\right)$.

Proof of lemma 7.6.ii. Let $\beta$ given by lemma 2.2 .

A conditioning on $\left\{K_{00}^{\varepsilon}, K_{01}^{\varepsilon}, K_{10}^{\varepsilon}\right\}$ yields

$$
\begin{aligned}
& E \exp (1+\beta) K\left(g_{1}\right)=E \Lambda_{\varepsilon} \exp (1+\beta) K_{00}^{\varepsilon}<\infty \\
& \Lambda_{\varepsilon}=\mu_{\varepsilon} \iint \exp (1+\beta)\left(\sum_{1 \leq j+k \leq 2} K_{j k}^{\varepsilon} y^{k} z^{j}\right) \exp \left(-\frac{z^{2}}{2 \xi_{0}^{T^{\prime}(\varepsilon)}}-\frac{(y-z)^{2}}{2 \xi_{T^{\prime}(\varepsilon)}^{T}}\right) d y d z .
\end{aligned}
$$

Since the deterministic $K_{02}^{\varepsilon}, K_{11}^{\varepsilon}, K_{20}^{\varepsilon}$ are bounded uniformly in $\varepsilon$,

$$
\sum_{j+k=2} K_{j k}^{\varepsilon} y^{k} z^{j} \geq-\operatorname{cst}\left(z^{2}+|y z|+y^{2}\right) \geq-\operatorname{cst}\left(z^{2}+(y-z)^{2}\right) .
$$


It yields

$\Lambda_{\varepsilon} \geq \mu_{\varepsilon} \iint \exp (1+\beta)\left(K_{01} y+K_{02} z\right) \exp \left(-c s t z^{2}-(y-z)^{2}\left(\xi_{T^{\prime}(\varepsilon)}^{T}\right)^{-1}\right) d y d z$.

If $X$ is a one-dimensional gaussian centered r.v., then $E \exp \lambda X \geq 1$ for all $\lambda \in \mathbb{R}$. Hence, there exists $m_{0}>0$ such that $\inf _{\varepsilon} \Lambda_{\varepsilon}>m_{0}$ a.s., and $\sup E \exp (1+\beta) K_{00}^{\varepsilon}<\infty$.

Proof of lemma 7.6.iii and 7.6.iv. Let $\nu \in] 1-d / 2+\gamma, 1+a / 2-d[$. Since $|v| \leq \lambda_{\varepsilon}^{-1} \varepsilon^{d / 2-\gamma}$, we have $\lambda_{\varepsilon} v=O\left(\varepsilon^{1-\nu}\right)$ and prop. 6.1 yields $g^{\varepsilon}\left(u, \lambda_{\varepsilon} v\right)$ tends to $g(u)$. Assume $\omega \in \cap_{\varepsilon} D_{\varepsilon} Q_{\varepsilon}$ (countable intersection, see remark 6.1). Assume $-\varepsilon^{-\gamma} \leq u \leq 0,1 \leq|v| \leq \lambda_{\varepsilon}^{-1} \varepsilon^{d / 2-\gamma}$. Lemma 7.5 yields

$$
\begin{aligned}
& \left|\sum_{1 \leq j+k \leq 2} K_{j k}^{\varepsilon} \Phi_{\varepsilon}^{Q}\left(\varepsilon u, \lambda_{\varepsilon} v\right)^{k} \Psi_{\varepsilon}^{Q}\left(\varepsilon u, \lambda_{\varepsilon} v\right)^{j}\right| \leq \varepsilon^{\gamma} \\
& -\frac{\left(\Phi_{\varepsilon}^{Q}\left(\varepsilon u, \lambda_{\varepsilon} v\right)-\Psi_{\varepsilon}^{Q}\left(\varepsilon u, \lambda_{\varepsilon} v\right)\right)^{2}}{2 \xi_{T^{\prime}(\varepsilon)}^{T}} \leq-\frac{1}{3} v^{2} \\
& \frac{\left(\Phi_{\varepsilon}^{Q}\left(\varepsilon u, \lambda_{\varepsilon} v\right)-\Psi_{\varepsilon}^{Q}\left(\varepsilon u, \lambda_{\varepsilon} v\right)\right)^{2}}{2 \xi_{T^{\prime}(\varepsilon)}^{T}} \longrightarrow \frac{1}{2} v^{2} \\
& \frac{\Psi_{\varepsilon}^{Q}\left(\varepsilon u, \lambda_{\varepsilon} v\right)^{2}}{2 \xi_{0}^{T^{\prime}(\varepsilon)}} \longrightarrow 0
\end{aligned}
$$

and we can conclude easily.

\section{APPENDIX}

Appendix 8.1. There exists $C>0$ such that, for all $\varepsilon, t, x$

$$
\begin{aligned}
\left|\partial_{x} u^{\varepsilon}\left(\varepsilon^{2} t, \varepsilon^{2} x\right)\right| & \leq C\left(t^{-1 / 2}+t^{1 / 2}|x|+t\right) \\
\left|\partial_{x x}^{2} u^{\varepsilon}\left(\varepsilon^{2} t, \varepsilon^{2} x\right)\right| & \leq C\left(t^{-1 / 2}+t^{2} x^{2}+t^{5 / 2}\right) .
\end{aligned}
$$

Proof. A similar result is known if $\sigma$ and $c$ are constant (see Uchiyama (1978)), and we use the same line of proof. Let $f(u)=1_{\{0 \leq u \leq 1\}} u(1-r(u))$.

Let $p_{t}(x, y)$ be the transition density of diffusion $d \bar{X}_{s}=\sigma\left(X_{s}\right) d W_{s}$. Then, the following problems 1 and 2 both have a unique solution, and they coincide.

Problem 1

$\partial_{t} u=\frac{1}{2} \sigma^{2}(x) \partial_{x x}^{2} u+c(x) f(u)$ if $t>0$

$\lim _{t \rightarrow 0} u(t, x)=1_{\{x \leq 0\}}$ uniformly on every compact of $\mathbb{R}^{*}$.

$u \in C^{1,2}(] 0,+\infty[\times \mathbb{R},[0,1])$

Problem 2

$u(t, x)=\int_{-\infty}^{0} p_{t}(x, y) d y+\int_{0}^{t} d s \int_{-\infty}^{+\infty} p_{t-s}(x, y) c(y) f(u(s, y)) d y$ if $t>0$ 
$u(0, x)=1_{\{x \leq 0\}}$

$u \in C(] 0,+\infty[\times \mathbb{R},[0,1])$

The proof of this equivalence is known if $c$ is bounded (see Bramson (1982)) and it can be extended when $c$ has, at most, a linear growth by using classical arguments (Feynman-Kac formula, maximum principle ...). We get

$$
u^{\varepsilon}\left(\varepsilon^{2} t, \varepsilon^{2} x\right)=\int_{-\infty}^{0} p_{t}^{\varepsilon}(x, y) d y+\int_{0}^{t} d s \int_{-\infty}^{+\infty} p_{t-s}^{\varepsilon}(x, y) c\left(\varepsilon^{2} y\right) f\left(u^{\varepsilon}\left(\varepsilon^{2} s, \varepsilon^{2} y\right)\right) d y
$$

where $p^{\varepsilon}$ is the transition density of the diffusion $d X_{s}^{\varepsilon}=\sigma\left(\varepsilon^{2} X_{s}^{\varepsilon}\right) d W_{s}$.

Since $\|\sigma\|_{\infty}<\infty$ and sup $\| \partial_{x} \sigma\left(\varepsilon^{2}\right.$. $) \|_{\infty}<\infty$, Friedman's estimates yield (see Friedman(1964)): $\begin{aligned} & \varepsilon \leq \varepsilon_{0} \\ & \exists C_{1}, C_{2}>0 \forall \varepsilon, t, x, y\end{aligned}$

$$
\begin{aligned}
& p_{t}^{\varepsilon}(x, y) \leq C_{1}(2 \pi t)^{-1 / 2} \exp \left[-C_{2}(x-y)^{2}(2 t)^{-1}\right] \\
& \left|\partial_{x} p_{t}^{\varepsilon}(x, y)\right| \leq C_{1}\left(2 \pi t^{2}\right)^{-1 / 2} \exp \left[-C_{2}(x-y)^{2}(2 t)^{-1}\right] \\
& \left|\partial_{y} p_{t}^{\varepsilon}(x, y)\right| \leq C_{1}\left(2 \pi t^{2}\right)^{-1 / 2} \exp \left[-C_{2}(x-y)^{2}(2 t)^{-1}\right] .
\end{aligned}
$$

Then, we get (8.1) by differentiating (8.3) and applying (8.4).

(8.2) cannot be derived by differentiating twice (8.3) (no dominated convergence). Let $A^{\varepsilon}$ and $\left(P_{s}^{\varepsilon}\right)_{s \geq 0}$ be the infinitesimal generator and the semi-group of $X^{\varepsilon}$.

Let $f_{s}^{\varepsilon}(x)=c\left(\varepsilon^{2} x\right) f\left(u^{\varepsilon}\left(\varepsilon^{2} s, \varepsilon^{2} x\right)\right)$ and $w^{\varepsilon}(t, x)=\int_{0}^{t} P_{t-s}^{\varepsilon} f_{s}^{\varepsilon}(x) d s$.

Then, since $A^{\varepsilon}$ and $P_{t-s}^{\varepsilon}$ commute,

$$
\begin{aligned}
& A^{\varepsilon} w^{\varepsilon}(t, x)=\int_{0}^{t} A^{\varepsilon} P_{t-s}^{\varepsilon} f_{s}^{\varepsilon}(x) d s=\int_{0}^{t} P_{t-s}^{\varepsilon} A^{\varepsilon} f_{s}^{\varepsilon}(x) d s \\
& =-\frac{1}{2} \int_{0}^{t} d s \int_{-\infty}^{+\infty} \partial_{y}\left[p_{t-s}^{\varepsilon}(x, y) \sigma^{2}\left(\varepsilon^{2} y\right)\right] \partial_{y}\left[c\left(\varepsilon^{2} y\right) f\left(u^{\varepsilon}\left(\varepsilon^{2} s, \varepsilon^{2} y\right)\right)\right] d y
\end{aligned}
$$

(last equality is a consequence of integration by part formula). (8.1), (8.4) and (8.6) imply $\left|A^{\varepsilon} w^{\varepsilon}(t, x)\right|=O\left(1+t^{2} x^{2}+t^{5 / 2}\right)$. It allows us to conclude since $\sigma \geq m>0$.

Appendix 8.2. Consider a brownian martingale $Z_{t}=x+\int_{0}^{t} A_{s} d W_{s}$ where A satisfies $\|A\|_{0}^{\infty} \leq M$ a.s. $(M \in \mathbb{R})$. Then

$$
\begin{aligned}
& \forall r>|x| \quad P\left(\|Z\|_{0}^{t} \geq r\right) \leq \exp -\frac{(r-x)^{2}}{2 t M^{2}}+\exp -\frac{(r+x)^{2}}{2 t M^{2}} \\
& \limsup _{s \rightarrow+\infty} \frac{\left|Z_{s}\right|}{\sqrt{2 s \log \log s}} \leq \frac{1+M^{2}}{2} \text { a.s. }
\end{aligned}
$$

Proof. (8.7) is a classical consequence of Doob inequality. Concerning (8.8), we copy the proof of the law of iterated logarithm for the brownian motion (see for instance Revuz and Yor (1991)). 


\section{ACKNOWLEDGEMENTS}

I would like to thank Alain Rouault for introducing me to the KPP equation and for his help during the making of this paper.

\section{ReFERENCES}

Athreya, K. and Ney, P. (1972). Branching processes, Berlin Heidelberg New York: Springer.

AzencotT, R. (1980-1981), Formule de Taylor stochastique et développements asymptotiques d'intégrales de Feynman, séminaire de probabilités XVI, Lecture Notes in Maths 921 237-284.

Azencott, R. (1985), Petites perturbations aléatoires des systèmes dynamiques: développements asymptotiques, Bulletin des sc. math. 2ème série, 109 253-308.

Ben Arous, G. (1988), Méthodes de Laplace et de la phase stationnaire sur l'espace de Wiener, Stochastics 25 125-153.

Ben Arous, G. and Rounult, A. (1993), Laplace asymptotics for reaction-diffusion equations, Probab. Th. Rel. Fields 97 259-285.

Bramson, M. (1982), The Kolmogorov non-linear diffusion equation, Lecture at Paris VI university.

Barles, G. and Souganidis, P.E. (1994). A remark on the asymptotic behavior of the solution of the KPP equation, C. R. Acad. Sci. Paris 319, Série 1 679-684.

Chauvin, B. and Rounult, A. (1988), KPP equation and supercritical branching brownian motion in the subcritical speed area: application to spatial trees, Probab. Th. Rel. Fields 80 299-314.

Dacunha-Castelle, D. and Florens-Zmirou, D. (1986), Estimation of the coefficients of a diffusion from discrete observations. Stochastics 19 263-284.

Doss, H. (1980), Quelques formules asymptotiques pour les petites perturbations de systèmes dynamiques, Ann. IHP XVI n ${ }^{\circ} 1$ 17-28.

Fitzsimmons, P., Pitman, J. and Yor, M. (1992), Markovian bridges: construction, Palm interpretation, and splicing, Cinlar, E. (ed) et al., Seminar on stochastic processes held at the univ. of Washington, DC, USA; prog. probab. 33 101-134.

Freiduln, M. (1985), Functional integration and partial differential equation, Princeton univ. press.

FreidLin, M. (1990), Semi-linear pde's and limit theorems for large deviations; école d'été de Saint-Flour. Lecture Notes in Maths 1527.

Friedman, A. (1964), Partial differential equations of parabolic type, Prentice Hall. Englewood Cliffs, N.J.

Kolmogorov, A., Petrovskit, I. and Piscunov, N. (1937), Etude de l'équation de la diffusion avec croissance de la quantité de matière et son application à un problème biologique, Moscow univ. bull. math. 11-25.

Kuo, H.H. (1975), Gaussian measure in Banach spaces, Lect. Notes in Math. 463.

Nualart, D. and Pardoux, E. (1988), Stochastic calculus with anticipating integrands, Probab. Th. Rel. Fields 78 535-581.

Revuz D. and Yor M. (1991), Continuous martingales and Brownian motion, SpringerVerlag Berlin Heidelberg.

UсhiYama, K. (1978), The behaviour of solutions of some non-linear diffusion equations for large time, Journ. math. Kyoto univ. 18 453-508.

Université de Versailles-Saint-Quentin, F-78035 Versailles, France.

EMAIL:rouques@math.uvsq.fr. 